INRA Prod. Anim., 2007, 20 (4), 275-294

\section{Superovulation chez la jument avec les hormones gonadotropes : le point sur la situation et nouvelles données}

\begin{abstract}
C. BRIANT ${ }^{1}$, D. GUILLAUME ${ }^{1}$, P.-L. TOUTAIN ${ }^{2}$, M.-R. BLANC ${ }^{1}$
${ }^{1}$ INRA, CNRS, Université de Tours, Haras Nationaux, UMR85 Physiologie de la Reproduction et des Comportements, F-37380 Nouzilly, France

2 INRA, Ecole Nationale Vétérinaire, UMR181 Physiopathologie et Toxicologie Expérimentales, F-31076 Toulouse, France

Courriel : briant@tours.inra.fr
\end{abstract}

\begin{abstract}
Dans l'espèce équine, les traitements de superovulation avec la eFSH sont uniquement utilisés au plan expérimental. Des posologies d'administration de 1'hormone inadaptées apparaissent comme responsables de la grande variabilité observée dans les réponses des juments traitées. Des études récentes ont déterminé cette posologie en fonction des taux de sécrétion endogènes de l'hormone.
\end{abstract}

Dans les conditions naturelles de reproduction, la jument donne naissance à un poulain par an, conséquence de la régulation naturelle du taux d'ovulation par cycle, limité à 1 dans cette espèce. Toutefois et plus fréquemment dans certaines races, des ovulations multiples sont rapportées. Il s'agit en général de doubles ovulations et dans un faible pourcentage de cas, de triples ou de quadruples ovulations. Malheureusement les gestations multiples qui en résultent ne sont pas souhaitées, car elles parviennent rarement à terme ou, si elles y parviennent, les jumeaux sont le plus souvent non viables ou présentent un fort retard de croissance.

L'objectif des traitements de superovulation est d'augmenter dans des conditions contrôlées le taux naturel d'ovulations, afin de produire au cours d'un même cycle après fécondation in vivo, plusieurs embryons, qui seront ensuite collectés à 7 jours, puis transférés chez des femelles receveuses. Si ces traitements étaient disponibles pour l'espèce équine, ils pourraient apporter un bénéfice évident pour l'élevage. En effet, même s'il ne sont susceptibles d'intéresser qu'un nombre limité de femelles, ces traitements permettraient d'augmenter le nombre de poulains de haute valeur génétique, issus de juments qui mènent de front une carriè- re sportive et reproductrice, ou de juments âgées dont les produits ont déjà montré des aptitudes particulières. Dans le cas de juments présentant des problèmes de fertilité, ils pourraient être associés aux techniques de procréation assistée (transfert d'ovocytes ou transfert précoce d'embryons).

Cependant, actuellement, aucun traitement de superovulation ne dispose d'une Autorisation de Mise sur le Marché (AMM) pour la jument. Les raisons en sont d'une part, l'hétérogénéité et la non répétabilité des réponses des femelles donneuses aux traitements et d'autre part, le fait que chez la jument, seules les gonadotrophines hypophysaires équines sont efficaces pour induire une stimulation ovarienne. Il n'est donc pas possible d'utiliser les préparations commerciales employées chez les ruminants : gonadotrophine chorionique équine (eCG) ou FSH porcine $(\mathrm{pFSH})$. Les préparations hormonales utilisées chez la jument sont produites exclusivement pour les besoins de la recherche. Deux types de préparations sont obtenus à partir d'hypophyses équines collectées en abattoirs : 1) un extrait brut (EPE: Equine Pituitary Extract) (Guillou et Combarnous 1983) utilisé à la fois grâce à ses effets FSH et $\mathrm{LH}$, pour la superovulation $(3,5$ à $7,6 \%$ d'activité
FSH) et pour l'induction d'ovulation (8 à $28 \%$ d'activité LH) (Hofferer et al 1993), ou 2) une préparation nettement plus concentrée en FSH utilisée exclusivement pour la superovulation (eFSH). Elle est obtenue à partir de l'EPE et a essentiellement une activité FSH (7 à 12,5\%) mais aussi une activité LH non négligeable (1,5 à $3 \%)$ (Hofferer et al 1993). Leur voie d'administration est en pratique intramusculaire (I.M.). Chez la jument la phase folliculaire dure en moyenne 8 jours et la phase lutéale 14 jours. Le traitement de superovulation est commencé au début ou au cours du premier tiers de la phase folliculaire, quand le follicule dominant atteint 15 à $20 \mathrm{~mm}$. Il est ensuite administré quotidiennement jusqu'au stade préovulatoire, quand le follicule dominant atteint environ $35 \mathrm{~mm}$. A ce moment, l'ovulation est induite par une injection de LH exogène. En effet, dans cette espèce, du fait de la longueur et de la variabilité de la période d'oestrus (2 à 10 jours), ce traitement est indispensable pour synchroniser les ovulations multiples et placer au mieux l'insémination artificielle, par rapport au pic de $\mathrm{LH}$ et à l'ovulation. Pour cette induction, deux molécules à effet LH, disposent d'une AMM en France pour la jument : l'hormone chorionique humaine (hCG, Chorulon $^{\circledR}$, Intervet) et un analogue de 
GnRH, la buséréline $\left(\right.$ Receptal $^{\circledR}$, Intervet). Un autre analogue de $\mathrm{GnRH}$, le Deslorelin, administrable sous forme d'implants (Ovuplant ${ }^{\circledR}$ ) est disponible notamment aux Etats-Unis.

Cet article présente une revue de l'ensemble des résultats obtenus avec les traitements de superovulation utilisant les gonadotrophines chez la jument. Les différentes causes identifiées comme responsables de la grande variabilité des réponses sont décrites ainsi que, quand elles existent, les solutions retenues pour y remédier. Enfin, différentes stratégies sont proposées pour le suivi d'études prospectives destinées à améliorer les traitements. Nous n'envisageons dans cet article que la production d'embryons in vivo par les femelles donneuses et non pas la production de poulains après transfert chez des juments receveuses. Des références aux autres espèces de rente sont faites quand elles permettent d'expliquer certains points ou d'émettre des hypothèses. En effet, certaines améliorations ont pu être apportées sur la base des résultats obtenus dans ces autres espèces. Ainsi, dans l'espèce bovine, qui montre également une grande variabilité des réponses des femelles donneuses, le choix de l'hormone injectée, l'adaptation des posologies, la modification des ratios FSH/LH dans les préparations hormonales, ainsi que la sélection des femelles bonnes répondeuses ont permis de mettre au point des protocoles utilisables en association avec le transfert embryonnaire. Toutefois, dans l'espèce bovine, le contexte est très différent. La technique est utilisée en routine dans les programmes de sélection. L'objectif est, pour un animal ayant un fort potentiel génétique, de récolter un nombre maximal d'embryons transférables puis d'obtenir ultérieurement, à partir d'un taux de gestation voisin de $50 \%$ après transfert chez les receveuses, une augmentation significative des veaux nés d'une même femelle. Dans l'espèce équine, le transfert embryonnaire n'est pas associé actuellement à une stimulation ovarienne préalable.

\section{1 / Chez la jument, les réponses ovariennes aux traitements de superovula- tion présentent une grande variabilité}

La réponse ovarienne d'une femelle donneuse à un traitement de superovulation est mesurable par le nombre de follicules préovulatoires, le nombre d'ovulations et enfin le nombre d'embryons transférables. La variabilité des réponses est observable à chacun de ces niveaux.

La figure 1 présente une synthèse des résultats pour l'ensemble des expériences publiées à ce jour. Chaque donnée correspond au résultat moyen obtenu dans un lot traité. La répartition moyenne des résultats obtenus dans les lots témoins non traités correspondants est donnée à titre de comparaison. Il ne s'agit pas ici de comparer les performances des différents traitements administrés mais d'avoir une vue d'ensemble des réponses.

La figure 1A présente le nombre moyen d'ovulations/cycle en fonction du nombre moyen de follicules préovulatoires/cycle. Dans les lots témoins, une moyenne de 0,8 à 1,3 follicules préovulatoires aboutit à une moyenne pratiquement identique d'ovulations. Ce qui signifie que chez les femelles non traitées quasiment tous les follicules préovulatoires ovulent. Certaines juments ont spontanément des ovulations doubles. Dans les lots traités, le nombre moyen de follicules préovulatoires/cycle est très variable (en moyenne 1,3 à 6,7 par cycle). Dans certaines expériences des moyennes basses résultent d'animaux n'ayant pas répondu au traitement en ne produisant qu'un seul follicule préovulatoire. Ainsi, dans une étude rétrospective sur un troupeau de juments poneys Welsh, $23 \%$ des femelles traitées $(\mathrm{n}=39)$ n'ont pas répondu au traitement de stimulation (Briant 2004). Globalement l'augmentation du nombre de follicules préovulatoires s'accompagne d'une augmentation du nombre d'ovulations (en moyenne 0,7 à 5 par cycle). Cependant, dans un nombre significatif d'expériences, le nombre d'ovulations est inférieur au nombre de follicules préovulatoires. Le traitement de superovulation induit donc une perte d'ovulations par rapport au nombre de follicules préovulatoires. De plus les juments poneys produisent moins de follicules préovulatoires et moins d'ovulations que les juments.

La figure 1B présente le nombre moyen d'embryons/cycle en fonction du nombre moyen d'ovulations/cycle. Dans les lots témoins, une moyenne de 0,8 à 1,3 ovulations/cycle permet de produire une moyenne de 0,4 à 0,9 embryons. Dans les lots traités, le nombre moyen d'ovulations par cycle varie de 1,6 à 7,1 chez la jument et de 0,7 à
2,9 chez la jument de race poney. Le nombre maximum d'ovulations rapporté chez une jument est de 18 et chez une jument poney de 6 . Le nombre minimum d'ovulation est de 0 (juments ayant plusieurs follicules préovulatoires qui n'ovulent pas) ou de 1 (juments n'ayant qu'un seul follicule préovulatoire et une seule ovulation). Ce nombre moyen d'ovulations/cycle dans les lots traités correspond à un nombre moyen d'embryons par cycle (récoltés entre 7 et 8 jours après ovulation) compris entre 0 et $3,5(0,47$ à 3,5 chez la jument et 0 à 1,1 chez la jument poney). Le nombre maximum d'embryons rapporté chez une jument est de 10 et chez une jument poney de 4 . Le profil du nuage de points montre que le nombre moyen d'embryons augmente proportionnellement au nombre moyen d'ovulations. Cependant, les résultats sont très hétérogènes entre expériences et les juments poneys semblent produire moins d'ovulations et moins d'embryons que les juments. De plus, un nombre significatif d'expériences ne rapporte aucun bénéfice du traitement de stimulation par rapport aux cycles non traités, en terme de nombre moyen d'embryons récoltés et ce, quelle que soit la race (Douglas 1979, Hofferer et al 1991, Dippert et al 1992, Palmer et Hajmeli 1992, Remy et al 1997, Niswender et al 2003, Briant et al 2004b, Welsh et al 2006). D'une façon générale, le nombre d'embryons n'est corrélé à aucune variable (taux d'ovulations, présence ou absence de pic de LH, taux d'oestrogènes) (Briant 2004, Squires et al 2006).

La figure 1C présente le taux de fertilité par ovulation, soit le nombre moyen d'embryons/ovulation/cycle en fonction du nombre moyen d'ovulations/cycle. Alors que dans les lots témoins ce taux est compris entre 0,6 et 0,7 , il varie entre 0,2 et 0,68 dans les lots traités. Plusieurs auteurs rapportent une baisse importante de ce taux au cours des cycles traités (Douglas 1979, Hofferer et al 1991, Dippert et al 1992, Palmer et Hajmeli 1992, Remy et al 1997, Scoggin et al 2002, Niswender et al 2003, Briant 2004, Machado et al 2004, Welsh et al 2006). La fertilité par ovulation est donc pratiquement toujours inférieure en cas d'ovulations multiples.

En conclusion, une réponse satisfaisante inclurait : la production d'un nombre suffisant de follicules préovulatoires (au moins 3), l'ovulation de tous ces follicules et l'obtention d'embryons à partir d'une majorité de ces 
Figure 1. Synthèse de l'ensemble des résultats publiés sur la superovulation avec FSH dans l'espèce équine.
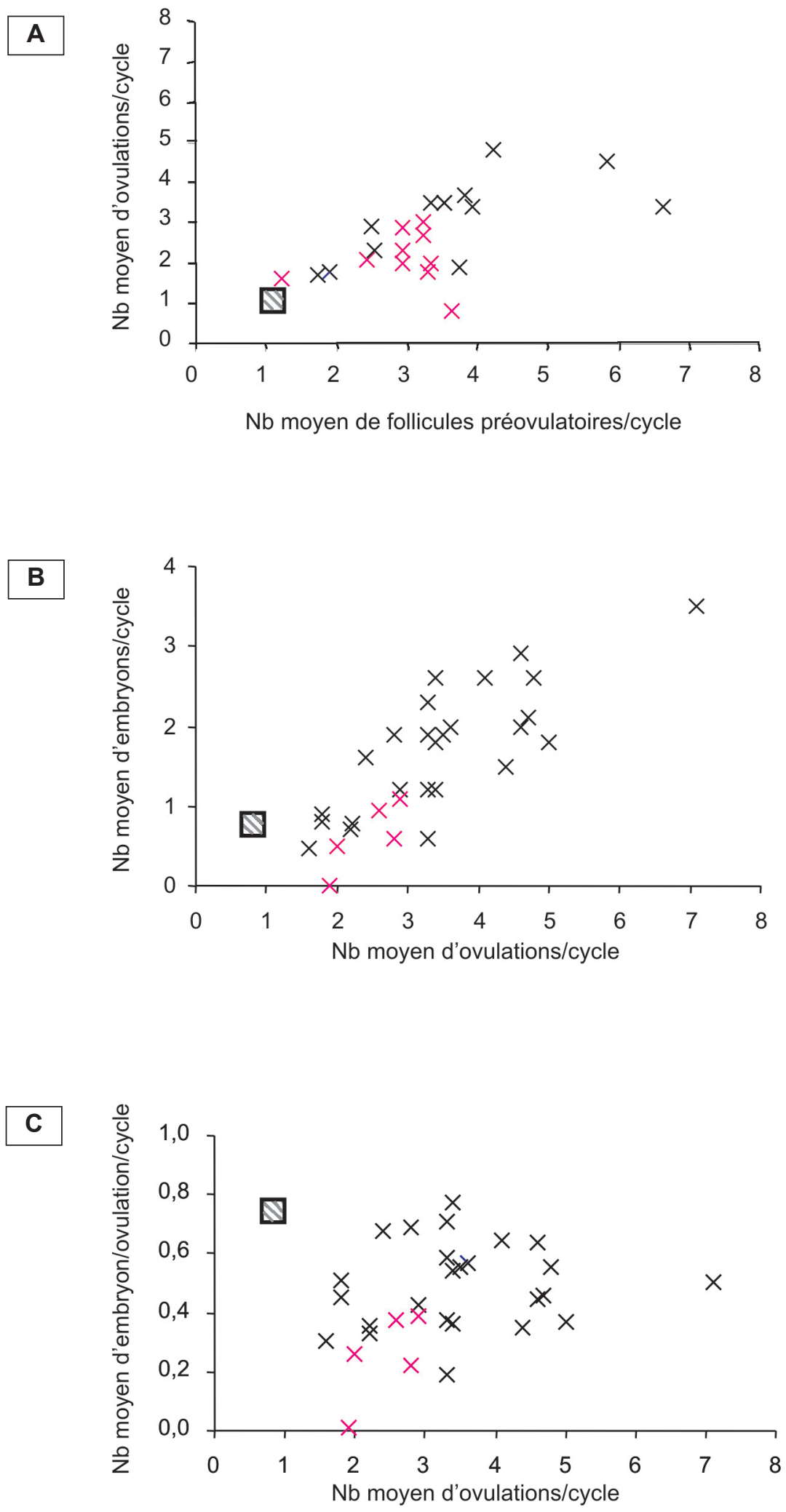

Chaque croix correspond au résultat obtenu dans chaque lot traité pour chaque expérience. Les croix noires représentent les données relatives aux juments et les croix rouges aux juments poneys. Les carrés hachurés représentent la plage de variation des résultats obtenus dans les lots témoins pour l'ensemble des expériences.

A : nombre moyen d'ovulations/cycle en fonction du nombre moyen de follicules préovulatoires/cycle,

B : nombre moyen d'embryons/cycle en fonction du nombre moyen d'ovulations/cycle,

C : nombre moyen d'embryon/ovulation/cycle en fonction du nombre moyen d'ovulations/cycle. ovulations. Même si une certaine «perte» est acceptable à chacun de ces trois niveaux, les paramètres nombre de follicules préovulatoires, nombre d'ovulations et nombre d'embryons devraient être corrélés. Les réponses non satisfaisantes résultent donc d'une perte anormale à un des ces trois niveaux.

De plus, contrairement à l'espèce bovine, où il semblerait que la «bonne» ou «mauvaise» réponse au traitement soit répétable pour une femelle donnée (Moor et al 1984), la répétabilité du facteur nombre d'embryons/cycle est faible chez la jument $(0,26)$ (Squires et al 2006). La sélection des femelles sur ce caractère, telle qu'elle est réalisée dans l'espèce bovine, apparaît donc moins justifiée. De plus, les juments susceptibles de bénéficier d'un traitement de superovulation associé à un transfert d'embryons sont des femelles de haute valeur génétique, de par leurs performances sportives ou celles de leurs produits. Donc, même si les chances d'obtenir un poulain sont faibles, tous les moyens seront mis en œuvre pour tenter d'y arriver.

\section{2 / Les causes de variabilité des réponses aux traite- ments de superovulation chez la jument sont multi- ples}

\section{1 / Variabilité du nombre de follicules préovulatoires.}

La production de follicules préovulatoires multiples est associée à une dose minimale de FSH bio disponible. Un exemple est donné par la figure 2 (Briant 2004). La mesure des concentrations plasmatiques de FSH chez des juments poneys Welsh traitées avec de la eFSH montre que l'administration d'une dose quotidienne de $2,2 \mathrm{mg}$ de eFSH par voie I.M. induit des taux à peine plus élevés que chez les animaux non traités. Chez certaines femelles traitées, les taux plasmatiques de FSH ne sont pas augmentés par rapport aux femelles non traitées. Ces taux relativement bas sont associés à une stimulation folliculaire insuffisante (1,3 follicules préovulatoires en moyenne), avec 2/3 des femelles ne répondant pas au traitement (un seul follicule préovulatoire). De plus, les femelles n'ayant pas répondu ne sont pas celles qui ont les taux les plus bas de FSH. Ceci suggère que la stimulation dépendrait d'une 
Figure 2. Profils plasmatiques moyens de FSH chez des juments non traitées $n=6$ ou traitées avec eFSH 2,2 mg/jour en 1 injection I.M. $n=6$ ou traitées avec eFSH $6,6 \mathrm{mg} / \mathrm{jour}$ en 3 injections I.V. $n=6$.

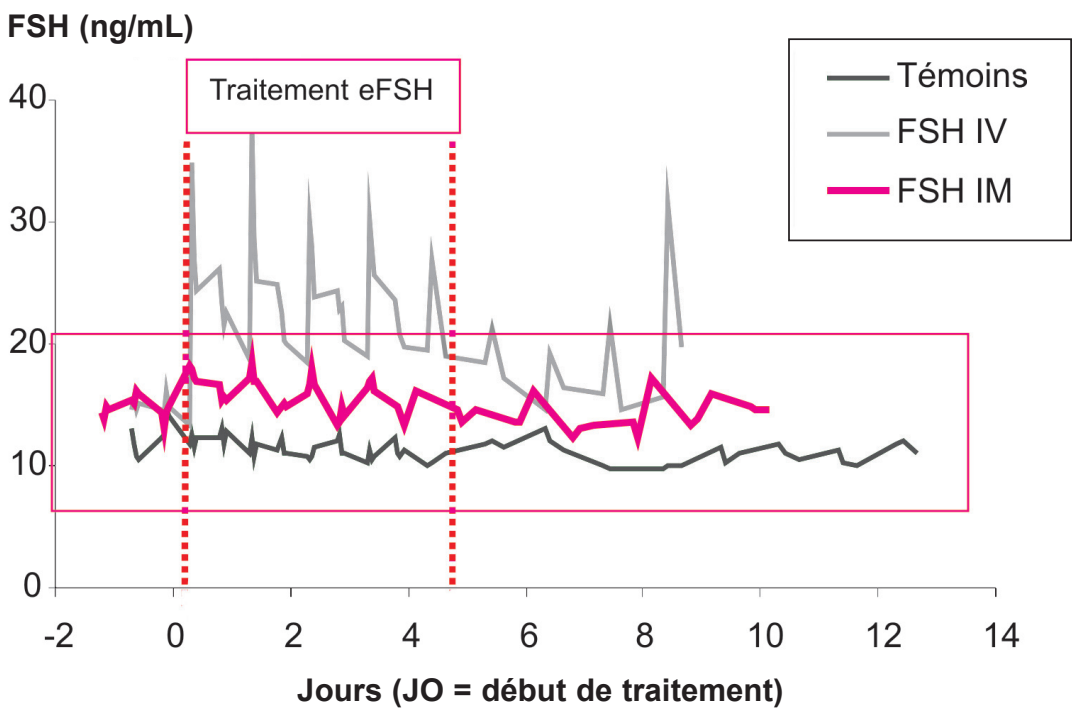

La zone encadrée en rouge représente la plage de variation des profils individuels pour les cycles traités par voie I.M. Les données sont présentées depuis le lendemain de l'injection de prostaglandine F $2 \alpha$ et J0 est le premier jour de traitement. La période de traitement est comprise entre les lignes pointillées rouges (adapté de Briant 2004).

dose seuil minimale individuelle. Des résultats assez comparables ont été obtenus chez la brebis, où il existe un seuil individuel de réponse à la $\mathrm{FSH}$, au dessus duquel chaque brebis présente une polyovulation. Ce seuil est égal à $125 \%$ des concentrations maximales de FSH au cours de la phase lutéale (Picton et McNeilly 1991).

$\mathrm{Au}$ contraire, des taux plasmatiques de FSH élevés sont induits par l'administration d'une forte dose de eFSH (3 injections quotidiennes de 2,2 mg par voie I.V.). Ces taux sont associés à un nombre satisfaisant de follicules préovulatoires (3,7 en moyenne) et toutes les femelles répondent au traitement par la production de 2 à 5 follicules préovulatoires.

La voie d'injection est très importante. En effet, la voie d'injection I.M. induit une variabilité importante des taux plasmatiques de FSH après injection. Au contraire, la voie I.V. permet une biodisponibilité maximale et une meilleure homogénéité entre femelles.

Mais l'augmentation du nombre de follicules préovulatoires n'est pas proportionnelle à la dose de FSH bio disponible. Dès lors qu'une stimulation folliculaire est observée (au moins 2 follicules préovulatoires), le nombre de follicules préovulatoires n'est pas corrélé aux taux moyens de FSH plasmatiques pendant le traitement et ce, quelle que soit la voie d'administration (I.M. ou I.V.) ou la dose (Briant 2004).
Enfin, le nombre maximum de follicules préovulatoires semble limité à un certain seuil. Chez la jument, le nombre moyen maximal de follicules préovulatoires observé dans un lot de juments traitées est de 6,7 (Niswender et al 2003) et dans un lot de juments poney de 3,7 (Briant 2004). Des doses croissantes de eFSH $(1,72,3,45$ et $6,6 \mathrm{mg}$ par jour), administrées à des juments poneys Welsh en 3 injections I.V. quotidiennes, n'augmentent pas significativement la stimulation folliculaire, puisque les nombres de follicules préovulatoires sont de 3,3, 3,4 et 3,7 respectivement dans chaque lot (Briant et al 2005). Il semble donc dans ce cas que la capacité de stimulation maximale de l'ovaire soit atteinte.

Le nombre de follicules préovulatoires dépendrait de facteurs génétiques et environnementaux. Chez la brebis, le rôle des facteurs d'origine génétique sur la réponse aux traitements de superovulation est connu depuis longtemps, puisque la réponse à eCG varie en fonction des races, indépendamment du poids (Bradford et al 1971). De plus dans cette espèce, les races avec les plus hauts taux d'ovulation naturelle sont également celles qui répondent aux doses plus faibles de gonadotrophines. Chez les bovins, il est reconnu que les lignées ayant un taux plus élevé d'ovulations naturelles (suggérant un support génétique, comme chez les ovins) ont également une meilleure réponse aux traitements de superovula- tion (Kafi et McGowan 1997). De plus, l'origine paternelle des donneuses est une source de variation pour toutes les variables de la réponse au traitement de superovulation (Ponsart et al 2004).

Chez les équins, la race semble avoir un effet significatif, puisque les juments ont des capacités de réponse à la stimulation supérieures aux juments poneys (Lapin et Ginther 1977, Douglas 1979, Palmer 1985, Briant 2004) avec un maximum rapporté de 18 ovulations chez une jument (Alvarenga et al 2001) et 6 ovulations chez une jument poney Welsh (Briant et al 2004b). Comme évoqué dans le paragraphe ci-dessus, à partir d'une certaine dose de FSH, l'augmentation de la dose injectée n'augmente plus la stimulation, qui a probablement atteint son maximum. Il est intéressant de rapprocher la capacité de stimulation inférieure des juments poneys à l'expression, également inférieure dans cette race, du taux de polyovulation naturelle. Ainsi, des taux de polyovulation naturelle de $0 \%$ à $11 \%$ ont été relevés chez les poneys (Ginther 1992) et plus particulièrement $5,9 \%$ dans une population de poneys Welsh (Briant 2004) et $10 \%$ dans une population de poneys Welsh et Shetland (Wesson et Ginther 1981). A titre de comparaison, des taux de 15 à $22 \%$ ont été rapportés chez des Pur-sang (Ginther et al 1982), de 14,5 (Osborne 1966) à 27,3 \% (Henry et al 1982) chez des juments de selle et de $17,5 \%$ chez des juments lourdes (David 1975).

Dans les espèces de rente, le rôle de différents facteurs environnementaux sur la réponse aux traitements de superovulation est connu. Ainsi, chez la vache le statut nutritionnel, l'état corporel, le passé reproducteur, l'âge, la saison, l'élevage (Mapletoft et al 2002), les conditions climatiques (Putney et al 1988, Hansen et al 2001) interviennent précocement et tout au long du processus de croissance folliculaire, notamment sur le nombre de follicules recrutés. Même si ces différents facteurs peuvent agir par des voies différentes, ils sont tous plus ou moins liés au statut nutritionnel et à l'état corporel. Ainsi, chez la vache, l'augmentation des apports nutritionnels permet d'augmenter la stimulation folliculaire. Dans cette espèce, l'augmentation de la ration de $100 \%$ par rapport aux besoins d'entretien, pendant une période de 3 semaines avant le traitement de $\mathrm{FSH}$, permet d'augmenter le nombre de petits follicules au début du traitement, le nombre de folli- 
cules de $9 \mathrm{~mm}$ de diamètre avant ovulation, et le nombre de corps jaunes $(18,1$ vs 10,6) (Gong et al 2002). Cependant, ce bénéfice n'est pas forcément répercuté sur le nombre d'embryons transférables, car le niveau de nutrition a également des répercussions sur la qualité de l'ovocyte. En effet, chez les ruminants, si des niveaux de nutrition élevés ont un effet bénéfique sur la qualité de l'ovocyte en cas d'ovulations spontanées, l'effet est plutôt défavorable chez les femelles superovulées (Robinson et al 2005).

Dans l'espèce équine, l'effet de ces facteurs sur la réponse aux traitements de superovulation n'est pas connu, mais il existe probablement. En effet, les mêmes facteurs que dans les autres espèces modulent le recrutement, au cours de cycles non stimulés. Ainsi, chez la jument et la jument poney le taux de polyovulation naturelle augmente avec l'âge (Ginther et al 1982, Ginther 1992, Briant et al 2005). Dans un troupeau de juments poneys Welsh il varie selon la saison, en étant maximal en pleine saison de reproduction soit d'avril à août, et augmente proportionnellement avec le poids des femelles (Briant et al 2005). Concernant plus particulièrement l'effet de 1'état corporel, chez la jument poney Welsh, une diminution de la ration stabilisée à $60 \%$ des besoins d'entretien, a des conséquences sur la croissance folliculaire. Cette restriction induit une diminution du volume total des follicules tout au long de la phase folliculaire, ce critère représentant à la fois le nombre de follicules et leur taille à un moment donné. $\mathrm{Au}$ moment de l'ovulation, le volume total moyen est de $50 \mathrm{~mL}$ chez les juments poneys grasses vs 25 à $30 \mathrm{~mL}$ chez les juments poneys maigres. De plus, la proportion de juments présentant des doubles ovulations spontanées est inférieure chez les juments maigres. Cet effet a pu être attribué à l'implication du système IGF (Guillaume et al 2006a).

\section{2 / Perte d'ovulations par rap- port au nombre de follicules préovulatoires et perte d'em- bryons par rapport au nombre d'ovulations.}

a) Les pertes d'ovulations et d'embryons sont attribuées en partie à des anomalies de la folliculogénèse associées à des perturbations endocrines.

Dans un certain nombre de cas, la stimulation ovarienne est satisfaisante et un nombre suffisant de follicules préovulatoires est obtenu. Mais, après administration du traitement d'induction d'ovulation, une partie seulement de ces follicules ovule normalement. Les autres follicules évoluent en structures atypiques. Dans d'autres cas, certains follicules ovulent avant d'atteindre la taille préovulatoire. Ces anomalies de la folliculogénèse réduisent donc le gain potentiel en embryons. Chez une même jument, certains follicules préovulatoires évoluent de façon atypique et d'autres ovulent normalement, sans toutefois produire d'embryon. Les anomalies de la folliculogénèse créent donc un environnement défavorable à la production d'embryons.

- Description des anomalies de la folliculogénèse

La figure 3 présente la chronologie d'apparition de ces structures au cours d'un cycle traité chez la jument. Elles ne sont pas observées chez toutes les femelles traitées. Toutes ou seulement une partie d'entre-elles peuvent être observées, au cours d'un même cycle, chez une femelle donnée.

Chez la jument, comme chez les autres espèces, la croissance folliculaire

Figure 3. Séquences des évènements associés au traitement de superovulation chez la jument.

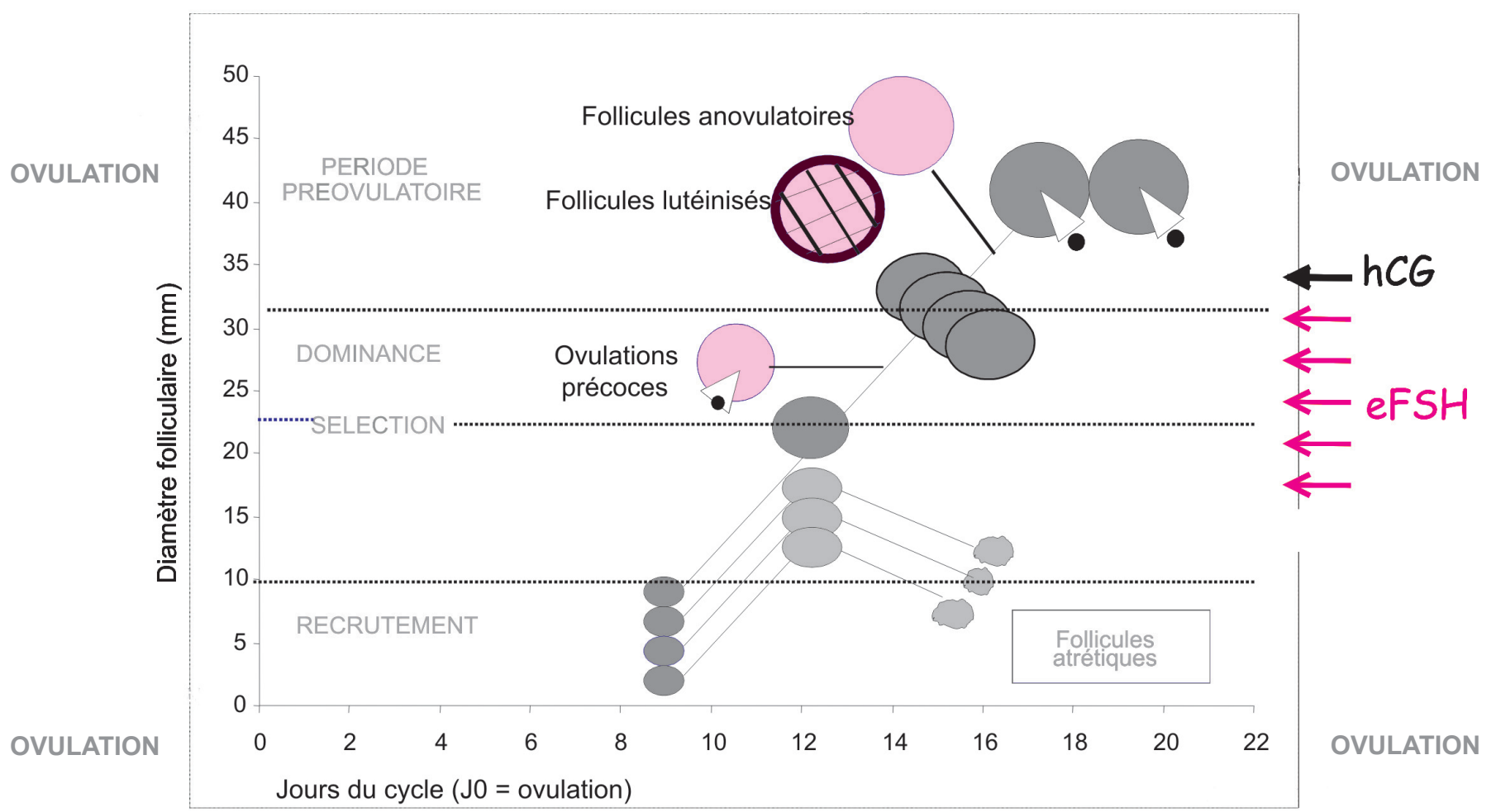

En gris évolution «normale» de la croissance folliculaire (Adapté de Ginther 1992 et Driancourt 2001). En rose, perturbations correspondantes (Adapté de Briant 2004).

Les flèches rouges et noires montrent les stades d'administration du traitement de superovulation avec eFSH et d'induction d'ovulation avec hCG. 
terminale comprend plusieurs phases, recrutement, sélection, dominance et période préovulatoire qui aboutit à l'ovulation. Les anomalies de la folliculogénèse peuvent apparaître dès le deuxième jour de traitement et jusqu'au jour de l'ovulation présumé.

Les ovulations précoces. Elles surviennent sur des follicules qui n'ont pas atteint la taille préovulatoire et dont le diamètre est compris entre 20 et $28 \mathrm{~mm}$. Leur incidence peut être importante ; au cours d'expériences ayant utilisé l'EPE, $33 \%$ des juments ont ovulé avant l'insémination artificielle (Dippert et al 1992) et $20 \%$ avant l'injection d'hCG (Scoggin et al 2002).

Les follicules lutéinisés. Au stade préovulatoire, certains follicules se transforment progressivement et partiellement en structures comparables à un corps jaune, sans que l'ovulation soit détectable à l'échographie. En fait, contrairement à une ovulation normale où le follicule se rompt, celui-ci se remplit progressivement de cellules qui sécrètent de la progestérone. Ces follicules surviennent dans 4 à $8 \%$ des cycles non stimulés (Ginther et Pierson 1989, Mc Cue et Squires 2002, Briant 2004). Chez la jument poney Welsh, ils concernent jusqu'à $20 \%$ des cycles en cas de traitement de superovulation alors que cette proportion est de 6,9\% dans le même troupeau au cours de cycles non stimulés (Briant 2004). Ils sont associés à une baisse de fertilité, car les taux de gestation de juments inséminées au cours de cycles avec follicules lutéinisés avoisinent $0 \%$, que les juments aient reçu un traitement de superovulation (Briant et al 2004b), ou non (Mc Cue et Squires 2002). Une jument qui a déjà présenté ce type de follicule a plus de chances de le présenter à nouveau au cours de la même saison de reproduction (Mc Cue et Squires 2002).

Les follicules anovulatoires. Certains follicules atteignent la taille préovulatoire, mais n'ovulent ni spontanément, ni en réponse à l'administration de $\mathrm{LH}$ exogène. Ils régressent progressivement et disparaissent en général au cycle suivant. Chez la jument poney Welsh, au cours d'une étude ayant regroupé 39 cycles traités par administration I.M. de eFSH ou d'EPE, un tiers des follicules préovulatoires n'a pas ovulé (Briant 2004). De même, d'autres études où la eFSH a été administrée par voie I.V. ont rapporté en moyenne 1,8 à 4,2 follicules anovulatoires/cycle
(Briant et al 2004b, Briant 2004). Cet effet semble bien être dû au traitement de superovulation et non à la polyovulation, car dans le même troupeau, une étude rétrospective sur les cycles avec polyovulation naturelle $(\mathrm{n}=314)$ a montré que tous les follicules préovulatoires ovulent (en moyenne 2,08/cycle) (Briant 2004).

\section{- Description des perturbations endo- crines}

Elles concernent les profils plasmatiques des gonadotrophines et des stéroïdes. La modification des profils plasmatiques de gonadotrophines a été décrite pour la première fois chez des juments ayant reçu un traitement d'EPE (1 dose quotidienne de $25 \mathrm{mg}$ par voie I.M.) (Palmer 1985).

La figure 4 présente les profils plasmatiques hormonaux moyens, obtenus chez des juments poneys Welsh, non stimulées ou traitées avec la eFSH, après induction de la croissance folliculaire par injection de prostaglandine $\mathrm{F} 2 \alpha$.

Chez les juments non stimulées, l'injection de prostaglandine induit une

Figure 4. Profils plasmatiques moyens des gonadotrophines et des stéroïdes. A) chez des juments non traitées $n=10$ (adapté de Briant et al 2003), B) chez des juments traitées avec eFSH 3,45 mg/jour en 3 injections I.V. $n=10$ (adapté de Briant 2004 et Briant et al 2004a).
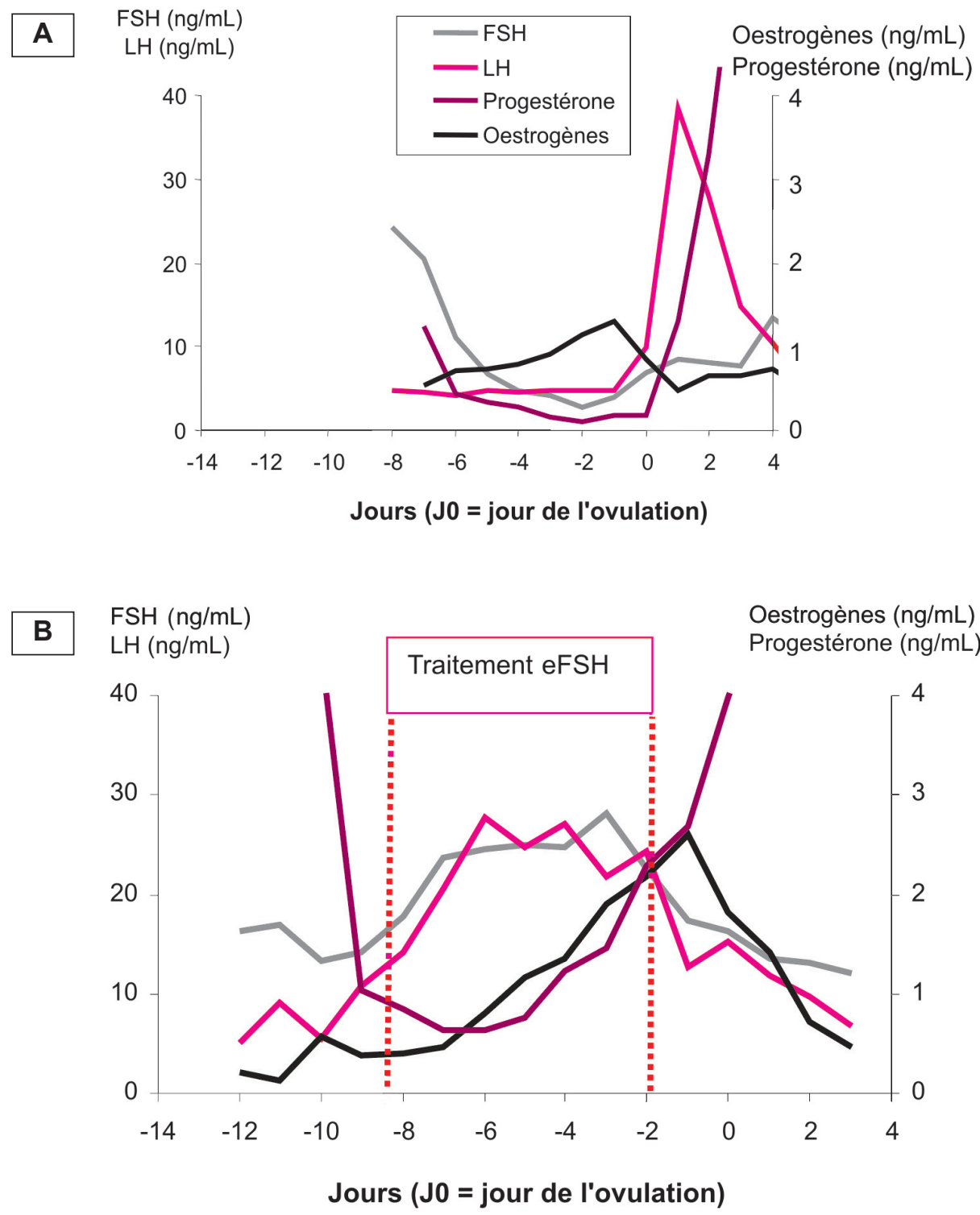

Les données sont présentées depuis le jour de l'injection de prostaglandine F2 $\alpha$ et J0 est le jour de l'ovulation. La période de traitement est comprise entre les lignes pointillées rouges. 
chute de progestérone qui permet la croissance d'une nouvelle vague folliculaire. Celle-ci est responsable de la montée progressive des oestrogènes totaux qui présentent un pic de l'ordre de $1 \mathrm{ng} / \mathrm{mL}, 1$ à 2 jours avant l'ovulation, puis rejoignent le niveau de base le lendemain de l'ovulation. Après injection de prostaglandine, les niveaux élevés de FSH permettant le recrutement baissent progressivement, du fait de l'effet de rétrocontrôle négatif exercé par le follicule dominant. A l'approche de l'ovulation, les taux de FSH restent bas, pour remonter au moment de l'ovulation et permettre le recrutement de la vague folliculaire du cycle suivant. Le pic périovulatoire de $\mathrm{LH}$, induit par la montée préovulatoire des oestrogènes s'amorce environ deux jours avant l'ovulation, atteint son maximum 1 à 2 jours après l'ovulation et se termine 4 à 5 jours après. Sa durée est donc très longue par rapport aux autres espèces domestiques de rente où il ne dure que quelques heures. Les concentrations de progestérone sont basses pendant toute la phase folliculaire. Elles augmentent le lendemain de l'ovulation, pour atteindre un plateau 5 jours plus tard et se maintiennent pendant toute la phase lutéale.

Chez les juments traitées, une augmentation importante des concentrations de FSH et de LH est observée, dès le début du traitement et pendant toute sa durée. Chez toutes les juments, les concentrations atteignent, pour la FSH les niveaux habituellement observés au cours de la phase lutéale et pour la LH, des concentrations équivalentes à celles d'un pic périovulatoire. A la même période, les niveaux d'oestrogènes totaux augmentent fortement et atteignent des concentrations voisines de 3 $\mathrm{ng} / \mathrm{mL}$, restent élevés plus longtemps qu'au cours de cycles non stimulés et ne retournent au niveau de base que 3 jours après l'ovulation. Chez $70 \%$ des juments, la progestérone présente des niveaux élevés ( $>1 \mathrm{ng} / \mathrm{mL}$ ) pendant toute la phase folliculaire et augmente de façon significative dès 4 jours avant l'ovulation. A l'arrêt du traitement, les concentrations de gonadotrophines baissent et la montée périovulatoire de LH n'est pas observée chez 80 $\%$ des femelles.

b) Les anomalies de la folliculogénèse et les pertes en embryons sont consécutives à une posologie d'administration des gonadotrophines inadaptée.

\section{- Observations doses/effets}

La posologie comprend l'association : 1) d'une dose totale quotidienne,
2) d'un nombre quotidien d'administrations, qui fractionne la dose totale au cours d'une journée ; le délai entre deux administrations est déterminé en fonction du temps de résidence de la molécule, afin de maintenir des concentrations efficaces, 3) d'une voie d'administration (orale, intraveineuse, intramusculaire..) qui peut modifier les doses réelles qui vont atteindre les organes cibles, après pertes dans différents tissus (muscle, graisse...).

Chez la jument (Scoggin et al 2002), l'administration d'une forte dose d'EPE (50 mg) en 1 seule injection quotidienne par voie I.M., augmente le nombre de follicules préovulatoires $(5,9)$ par rapport à une administration en 2 injections de $25 \mathrm{mg}(4,3)$. Mais, la dose de $50 \mathrm{mg}$ injectée en 1 fois, induit la formation de follicules anovulatoires et n'apporte aucun bénéfice en nombre d'ovulations, par rapport à la même dose administrée en 2 injections $(4,4 \mathrm{vs}$ 4,7). En parallèle, la dose totale de $25 \mathrm{mg}$ administrée en 2 injections de $12,5 \mathrm{mg}$ n'induit une stimulation que chez seulement 6 juments sur 10, avec en moyenne 3,6 follicules préovulatoires et 3,4 ovulations. Concernant la fertilité, c'est le lot recevant 2 injections de $12,5 \mathrm{mg}$ qui produit le plus d'embryons : en moyenne 2,6 embryons /cycle vs 1,2 et 1,5 embryons par cycle pour les lots $50 \mathrm{mg}$ en 1 injection et $50 \mathrm{mg}$ en 2 injections. De même le nombre d'embryon/ovulation est supérieur dans le lot recevant 2 injections de $12,5 \mathrm{mg}$ par rapport à tous les autres (0,76 vs 0,36 et 0,43 respectivement). Dans cette expérience le nombre moyen d'embryons le plus élevé a donc été obtenu dans le lot où le plus faible nombre de juments a répondu à la stimulation. Un résultat comparable a été obtenu avec l'administration de eFSH (Bioniche Canada), à la dose de 12,5 mg 2 fois par jour, soit une moyenne de 4,8 ovulations et 2,6 embryons (Machado et al 2004).

Les auteurs concluent que la posologie «idéale» pour optimiser la production d'embryons serait un compromis entre une dose suffisante pour permettre une stimulation ovarienne satisfaisante, mais pas trop forte pour éviter les effets indésirables (Scoggin et al 2002).

\section{- Processus impliqués}

Afin de comprendre les processus impliqués, des éléments de réponse ont été recherchés dans les autres espèces de rente, notamment les ruminants et plus particulièrement l'espèce bovine, où des effets comparables ont été décrits et en partie corrigés par des modifications des traitements.

Dans l'espèce bovine, une grande variabilité des réponses des femelles donneuses est rapportée. Par exemple, une étude sur plus de 2000 donneuses montre que, en moyenne, 11,5 embryons ont été obtenus par cycle, dont 6,2 transférables, mais $24 \%$ des collectes n'ont pas donné d'embryons viables, et $30 \%$ des collectes ont donné $70 \%$ des embryons transférables (Mapletoft et al 2002). La «bonne» ou «mauvaise» réponse au traitement serait répétable pour une femelle donnée (Moor et al 1984).

Des anomalies de la folliculogénèse et des modifications importantes des profils plasmatiques hormonaux semblables à celles observées chez la jument sont rapportées :

- augmentation des concentrations basales de FSH et de LH, lors d'administration de pFSH (Roberge et al 1995),

- augmentation importante et prolongée d'estradiol (Roberge et al 1995), plus marquée lors de l'administration d'eCG (Takagi et al 2001),

- sécrétion prématurée de progestérone avant le pic préovulatoire de $\mathrm{LH}$ à des taux supérieurs à $0,7 \mathrm{ng} / \mathrm{mL}$, ce qui témoigne de la présence d'un corps jaune actif (Jensen et al 1982).

- modifications quantitatives et temporelles du pic de LH avec :

soit une diminution de la sécrétion basale, de la fréquence et de l'amplitude des pulses de LH (Roberge et al 1995). Avec eCG (Saumande 1980) ou pFSH, le pic préovulatoire de LH est totalement supprimé, que les vaches aient subi ou non une synchronisation avec un progestagène (Nibart et al 1988, Humblot et al 1994). Dans certaines expériences, jusqu'à un quart d'entre elles ne présente pas de pic de LH (Humblot et al 1994),

. soit une avancée du pic préovulatoire de LH. Lors de traitement avec eCG, il survient $41 \mathrm{~h}$ après l'injection de PGF $2 \alpha$ chez les vaches traitées $v s 65 \mathrm{~h}$ chez les témoins (Jensen et al 1982). Après administration de FSH humaine recombinante cette durée est intermédiaire (47 h) (Takagi et al 2001).

Les relations suivantes ont été établies :

- l'augmentation importante et prolongée de l'oestradiol est attribuée aux gros follicules anovulatoires. Ces follicules sont associés à une stimulation excessive et sont plutôt observés avec 
l'eCG (Saumande 1980), mais on les trouve également avec la pFSH (Roberge et al 1995) ou la FSH recombinante humaine (Takagi et al 2001). L'association de l'anticorps anti-eCG, après le traitement par eCG, a permis de diminuer ces effets en réduisant le nombre total de follicules, le nombre de follicules anovulatoires et en augmentant le nombre d'embryons transférables (Gonzalez et al 1994) ;

- la sécrétion prématurée de progestérone est attribuée, soit à une stimulation de la sécrétion de progestérone du corps jaune actif avant l'administration de PGF2 $\alpha$ (Saumande 1980, Roberge et al 1995), soit à une sécrétion par des follicules lutéinisés (Saumande 1980, Jensen et al 1982), soit à la formation de nouveaux corps jaunes suite à des ovulations précoces (Jensen et al 1982). Des taux de progestérone anormalement élevés avant ovulation (plus de $1 \mathrm{ng} / \mathrm{mL}$ ), mesurés chez les vaches traitées avec eCG ou pFSH, sont associés à une réduction du nombre d'embryons récoltés ou transférables (Jensen et al 1982, Roberge et al 1995). Différents auteurs proposent que la modification des profils de stéroïdes associée aux traitements de superovulation pourrait interférer avec le transport des gamètes et des embryons. Ainsi, chez la brebis, les traitements de superovulation sont responsables d'une réduction du nombre de spermatozoïdes dans toutes les parties du tractus génital femelle, ainsi que d'une diminution du nombre de spermatozoïdes surnuméraires dans la zone pellucide chez la brebis et la vache (Greve et Callesen 2001). Or, au cours de cycles non stimulés, l'œstradiol et la progestérone agissent sur le stockage et la libération des spermatozoïdes dans l'oviducte, la contractilité de l'infundibulum et la formation du fluide de l'oviducte (Kafi et McGowan 1997). Plus particulièrement, la progestérone agit en modifiant les gradients de $\mathrm{Ca}++$ qui déclenchent la libération des spermatozoïdes, du réservoir de l'isthme vers le site de fécondation dans l'ampoule, 6 à $12 \mathrm{~h}$ après saillie ou IA. Au cours des traitements de superovulation, les modifications quantitatives et temporelles des sécrétions d'oestradiol et de progestérone pourraient donc perturber la rencontre entre l'ovocyte et les spermatozoïdes ;

- l'inhibition du pic de LH serait produite par un facteur ovarien sécrété par les nombreux follicules stimulés. Différents facteurs ont été proposés. Chez la vache, lors de traitement avec eCG, il s'agirait d'un effet combiné de la progestérone et de l'œstradiol sur la pulsatilité de GnRH, mais la progestérone ne serait pas le responsable majeur (Price et al 1998). De même, avec la pFSH, la progestérone (Humblot et al 1994) ainsi que des composés autres que l'œstradiol interviendraient (Takagi et al 2001), comme l'inhibine ou l'œstrone (Price 1995). Un autre facteur ovarien non stéroïde, le Gonadotropin-Surge Attenuating/ Inhibiting Factor (GnSAF/IF) a été identifié comme responsable d'une baisse de réponse hypophysaire à la GnRH après superovulation, chez la vache, la femme, la truie, la ratte (Fowler et al 2003). Il est différent de l'inhibine (Byrne et al 1995) ;

- la formation des follicules anovulatoires chez la vache serait en partie consécutive à l'absence de pic de LH (Nibart et al 1988, Humblot et al 1994). Afin de la compenser, l'administration de GnRH a permis une amélioration de la qualité des embryons (Foote et Ellington 1988). Cependant, d'autres causes sont probables car certains follicules n'ovulent pas, même après administration de LH ou d'une molécule à effet équivalent (Liu et Sirois 1998). Contrairement aux follicules «normaux» qui montrent une diminution de la sécrétion d'œstradiol et une augmentation de la progestérone 15 à $17 \mathrm{~h}$ après le pic de LH, les follicules anovulatoires contiennent encore de l'œstradiol $30 \mathrm{~h}$ après ce pic (Callesen et al 1986). Ce défaut d'ovulation a été associé à une absence d'expression de la prostaglandine $\mathrm{G} / \mathrm{H}$ synthase 2 (PGHS2), enzyme clé dans la synthèse des prostaglandines, induite dans les cellules de granulosa par le pic préovulatoire de LH endogène, ou par l'injection d'hCG (Liu et Sirois 1998). Celle-ci serait consécutive à une désensibilisation du follicule à la $\mathrm{LH}$, du fait d'un apport trop précoce de cette hormone. En effet, chez la ratte, une réduction du nombre des récepteurs à la $\mathrm{LH}$ est rapportée, après administration de LH en présence de follicules préovulatoires (Rao et al 1977) ;

- l'avancée du pic de LH est attribuée aux concentrations précoces et élevées d'œstradiol engendrées par la stimulation folliculaire, car les vaches traitées avec eCG ont des concentrations d'œstradiol 3 fois plus élevées (Takagi et al 2001). Les ovulations précoces en seraient la conséquence ;

- les altérations du pic de LH sont associées à des défauts de fécondation ou des pertes embryonnaires. Ainsi, parmi les vaches traitées, celles qui produisent des embryons de bonne qualité ont toutes des pics de LH détectables, au contraire de celles présentant des défauts de fécondation, qui n'ont pas de pic de LH (Jensen et al 1982) ou un pic avancé (Donaldson 1985). De plus, le nombre d'embryons transférables est corrélé aux concentrations maximales du pic de LH quand ce pic est situé au bon moment (Donaldson 1985). Dans l'espèce bovine, le moment du pic de LH par rapport au début de l'oestrus est particulièrement important, car l'insémination est effectuée par rapport à l'observation de l'oestrus et doit être la plus proche possible de l'ovulation. Ainsi, le nombre d'embryons viables et de bonne qualité est diminué quand l'intervalle entre le pic de LH et l'insémination est inférieur à $10 \mathrm{~h}$, ce qui induit un allongement de l'intervalle entre l'insémination et l'ovulation. Les traitements utilisant un dispositif intra vaginal de délivrance de progestérone permettent de mieux synchroniser l'occurrence du pic de LH par rapport à la fin du traitement de FSH (Lafri et al 2002).

Certains auteurs ont également proposé que les modifications des profils de la LH pourraient conduire à des anomalies de fécondation, du fait d'une altération de la qualité des ovocytes. En effet, la collecte d'ovocytes sur des vaches superovulées a montré, par rapport aux femelles non traitées un plus faible taux de récupération chez les vaches stimulées ainsi qu'une dégénérescence de l'ooplasme, une immaturité du fait d'une absence de pic, une asynchronie entre la maturation folliculaire et ovocytaire (Callesen et al 1986, Hyttel et al 1986, De Loos et al 1991). D'autres expériences, menées chez la brebis ont mis en évidence une activation nucléaire de l'ovocyte due à la montée trop précoce de LH. Ainsi, des ovocytes collectés sur des brebis traitées avec eCG présentent une plus forte proportion d'activation avec rupture de la vésicule germinale (33\%) que ceux issus de femelles non traitées $(0 \%)$ (Moor et al 1984). De même, après traitement de vaches avec l'extrait hypophysaire équin (EPE), les ovocytes contenus dans $77,5 \%$ des gros follicules non atrétiques sont activés, dont $64,5 \%$ au stade métaphase 1 (Moor et al 1984).

En conclusion, dans l'espèce bovine, malgré ces quelques interrogations persistantes, en 20 ans, un gain d'environ deux embryons viables par collecte a été observé, passant de 3,4 à 5,6 en moyenne, du fait principalement d'une diminution de la proportion de collectes sans embryons (Ponsart et al 2004). Les différentes étapes de l'amélioration 
ont été obtenues grâce au remplacement de la eCG par la FSH, les préparations étant plus pures et ayant un rapport FSH/LH plus stable, l'administration de l'hormone à doses décroissantes en deux injections quotidiennes, l'utilisation de traitements de synchronisation, et une meilleure maîtrise des facteurs de l'environnement, en particulier de l'alimentation.

Partant des modèles proposés dans l'espèce bovine, de nouvelles études ont été conduites chez la jument afin de déterminer les interactions entre les altérations de la folliculogénèse et les perturbations endocrines et de préciser quels étaient les paramètres agissant sur la production d'embryons. Il a ainsi été établi que, chez la jument poney Welsh (Briant 2004), l'administration de fortes doses de eFSH $(3,45 \mathrm{mg}$, en 3 injections quotidiennes, par voie I.V.), se traduit par des concentrations plasmatiques élevées de FSH et de LH. La LH résulte d'une purification insuffisante de la préparation de FSH. Ces concentrations élevées surviennent très précocement au cours de la phase folliculaire, quand le follicule dominant atteint à peine $20 \mathrm{~mm}$, alors que la taille préovulatoire est de l'ordre de $33 \mathrm{~mm}$. Elles sont à l'origine, soit des ovulations précoces, soit de la formation des follicules lutéinisés, qui induisent une augmentation des taux de progestérone, avant la date attendue de l'ovulation. En effet, les taux de LH pendant le traitement sont positivement corrélés aux taux de progestérone à cette même période, eux même corrélés au nombre de follicules lutéinisés. L'effet de la LH à ce stade est possible, car les récepteurs à la LH sont présents dès que le follicule atteint $20 \mathrm{~mm}$ et l'administration d'EPE, en présence de follicules de $20 \mathrm{~mm}$ de diamètre, induit une synthèse de progestérone (Brück et al 2000). Dans les deux cas, ovulation ou lutéinisation, le follicule est capable de produire de la progestérone mesurable dans le plasma, dès 2 jours après le début du traitement, soit 4 jours avant l'ovulation prévue. De plus, chez les juments présentant des follicules lutéinisés, les concentrations maximales d'oestrogènes totaux $(\mathrm{J}-1$ ou $\mathrm{J}-2$ par rapport à l'ovulation) et les concentrations de progestérone avant l'ovulation (J-3 à $\mathrm{J} 0)$ sont négativement corrélées (Briant 2004). La sécrétion anormale de progestérone avant ovulation s'accompagne donc d'une réduction relative de la sécrétion des œstrogènes. Ce type d'observation n'a jusqu'à présent été rapporté dans aucune espèce. Ces deux hormones étant sécrétés par les mêmes cellules, l'excès de LH en milieu de phase folliculaire induit donc une déviation de la stéroïdogénèse au niveau des cellules de la granulosa.

Ensuite, à l'arrêt du traitement avec eFSH, le pic endogène périovulatoire de LH est déprimé, voire supprimé. Comme chez la vache, cette inhibition est associée à l'augmentation des œstrogènes produite par le traitement. En effet, quelle que soit la dose de eFSH injectée $(1,72,3,45$ ou $6,6 \mathrm{mg}$ par jour en trois injections I.V. quotidiennes), les concentrations moyennes de $\mathrm{LH}$ au cours du pic périovulatoire sont négativement corrélées, soit aux concentrations d'œstrogènes deux jours avant ovulation, soit au nombre de follicules de plus de $20 \mathrm{~mm}$, soit au nombre de follicules préovulatoires (Briant 2004). Comme chez la vache, l'implication d'un facteur ovarien sécrété par les nombreux follicules stimulés peut être proposée, tel l'oestradiol, l'inhibine ou le GnSAF/IF. Par contre, contrairement à l'espèce bovine, il ne semble pas que la progestérone sécrétée avant ovulation ait un rôle dans cette inhibition. En effet : 1) chez des juments poneys Welsh traitées quotidiennement avec hCG (1600 UI), et ayant produit des follicules lutéinisés, la progestérone plasmatique augmente avant ovulation, sans que le pic périovulatoire de LH soit déprimé, 2) au contraire, après traitement de superovulation avec eFSH, la suppression du pic de LH peut survenir sans sécrétion précoce de progestérone (Briant 2004).

Une étude a tenté de déterminer l'implication respective d'un excès de FSH et/ou de LH, dans la survenue des follicules lutéinisés ou anovulatoires, des ovulations précoces et des pertes embryonnaires (Briant 2004). Au cours de celle-ci, l'hormone hCG, qui a un effet LH chez la jument, a été administrée quotidiennement à des juments poneys Welsh, pendant 5 jours, à la période où le traitement de superovulation est habituellement appliqué (1 ère injection en présence d'un follicule dominant de $20 \mathrm{~mm}$ ). La dose quotidienne (1600 UI par voie I.V.) était équivalente à celle qui permet d'induire l'ovulation, quand elle est administrée en injection unique en présence d'un follicule préovulatoire. Deux autres lots de femelles ont été observés en parallèle : un lot traité avec eFSH $(3,45 \mathrm{mg}$ en trois injections I.V. quotidiennes) et un lot témoin ne recevant pas d'injection. La figure 5 présente les profils hormo- naux plasmatiques moyens pour les trois lots.

L'administration quotidienne d'hCG induit une légère stimulation folliculaire puisque $50 \%$ des juments présentent au moins deux follicules préovulatoires. Ce lot est intermédiaire entre le lot non traité $(10 \%)$ et le lot recevant eFSH $(90 \%)$. Par contre, le nombre moyen d'ovulations n'est pas augmenté dans le lot ayant reçu l'hCG $(1,1)$ par rapport au lot témoin (1). Concernant le pourcentage de cycles avec follicules lutéinisés, le lot hCG avec $30 \%$ des cycles est également intermédiaire entre le lot non traité $(0 \%)$ et le lot recevant eFSH $(70 \%)$. Chez les juments traitées avec hCG et présentant des follicules lutéinisés, la sécrétion de progestérone est détectable au moins deux jours avant l'ovulation, alors que le pic préovulatoire d'oestrogènes est supprimé. Chez ces juments, la sécrétion de progestérone s'effectue donc au détriment de celle des œstrogènes. L'excès d'une activité LH au milieu de la phase folliculaire a donc activé précocement le processus de lutéinisation des cellules de la granulosa. Le pourcentage de cycles avec ovulation précoce tend à augmenter entre les cycles non traités $(10 \%)$, traités avec eFSH $(20 \%)$ et traités avec hCG $(50 \%)$. Le pourcentage de cycles avec formation de follicules anovulatoires est supérieur dans le lot traité avec eFSH $(60 \%)$ par rapport au lot témoin $(0 \%)$, le lot recevant hCG étant intermédiaire $(10 \%)$. Enfin, l'injection d'hCG semble produire une stimulation de la sécrétion endogène de LH (dans le dosage radio immunologique de la eLH, l'anticorps ne reconnaît pas hCG). Cette observation a été confirmée récemment (Evans et al 2006), aucun mécanisme d'action n'a été proposé, mais les auteurs émettent l'hypothèse que cette montée de $\mathrm{LH}$ endogène, suite à l'injection d'hCG, contribue peut être à l'effet d'hCG dans l'induction d'ovulation chez la jument. Il ressort donc de cette expérience qu'une activité LH prédominante seule est capable d'induire une stimulation folliculaire modérée, des ovulations précoces, la formation de follicules lutéinisés mais pas la formation de follicules anovulatoires.

Les mécanismes de formation des follicules anovulatoires restent donc difficiles à expliquer. Il est clair qu'ils sont associés à une hyperstimulation, induite par l'association $\mathrm{FSH} / \mathrm{LH}$, induisant des concentrations élevées d'oestrogènes. Contrairement à la vache, l'hypothèse d'une conséquence 
Figure 5. Profils plasmatiques moyens $(A)$ de $L H$, (B) d'oestrogènes totaux et $(C)$ de progestérone, chez des juments non traitées $n=10$, traitées avec eFSH 3,45 mg/jour en 3 injections I.V. $n=10$, ou traitées avec hCG 1600 Ul/jour I.V. $n=10$ (Briant 2004).

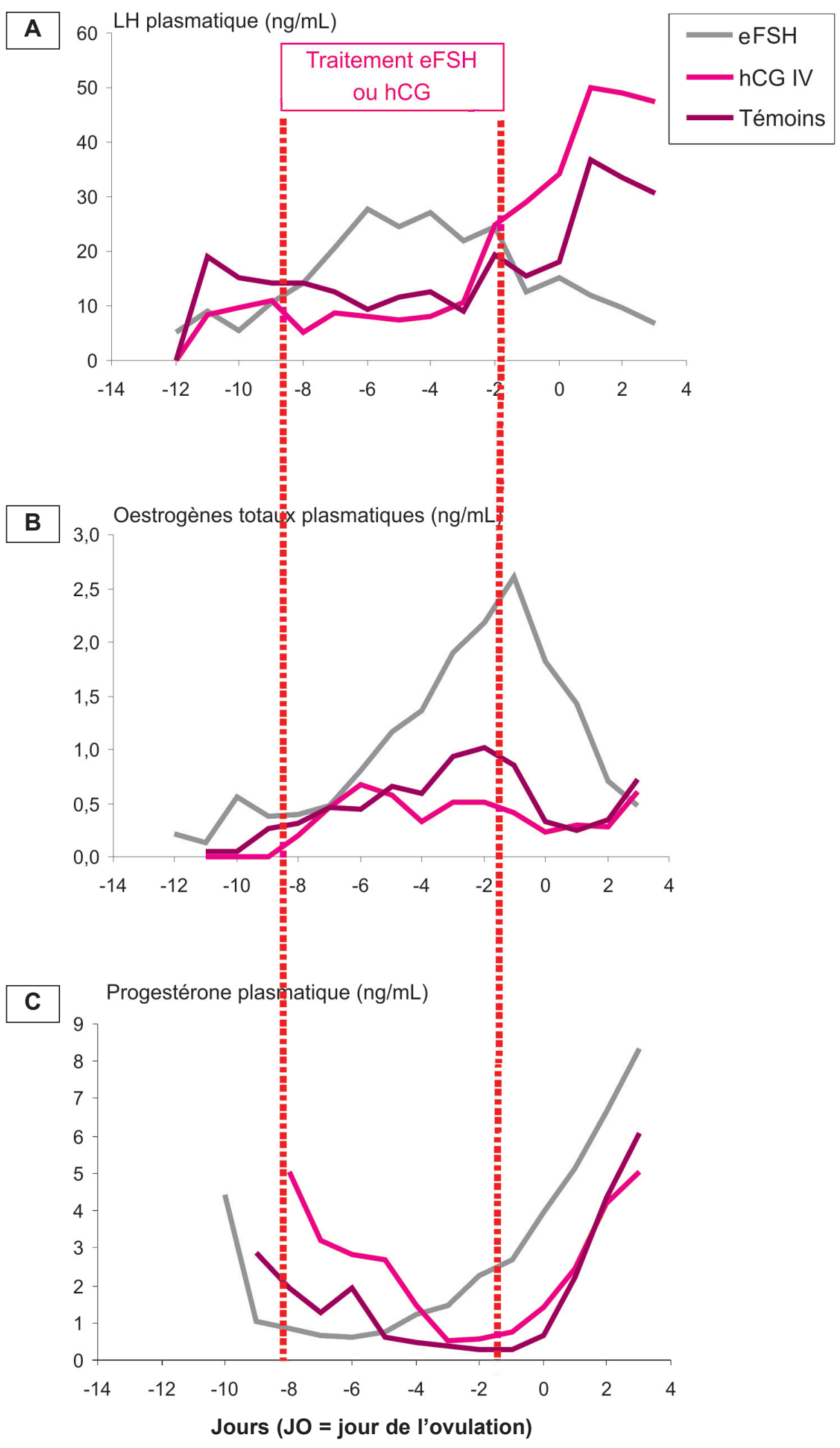

Les données sont présentées depuis le jour de l'injection de prostaglandine F2 $\alpha$ et J0 est le jour de l'ovulation. La période de traitement est comprise entre les lignes pointillées rouges.

de l'absence de pic de LH, suite aux concentrations élevées d'oestrogènes est peu probable, car dans l'espèce équine le traitement avec eFSH est toujours associé à une injection ovulante d'hCG, lorsque la majorité des follicu- les a atteint la taille préovulatoire. Ce raisonnement n'est cependant pas valable si l'on conçoit que l'ovulation induite par hCG résulte en partie d'une stimulation de la LH endogène. Ceci permettrait également d'expliquer, pourquoi l'hCG est plus efficace qu'un analogue de GnRH (Deslorelin, Ovuplant ${ }^{\circledR}$ ), pour induire les ovulations après traitement de superovulation (Niswender et al 2003).

La seconde hypothèse proposée chez la vache et relative à une non réponse à hCG, du fait d'une désensibilisation des récepteurs folliculaires à la $\mathrm{LH}$, peut être envisagée. Cependant, chez la jument, l'apport quotidien et répété d'hCG seule, à dose ovulante, pendant 5 jours consécutifs (voir ci-dessus) n'entraine pas la formation de follicules anovulatoires. Les explications potentielles pourraient être des activités différentes de l'hCG et de la eLH endogène au niveau folliculaire. Certains auteurs ont tenté de limiter cette potentielle désensibilisation des récepteurs ovariens, en observant une période de repos de 42 à $54 \mathrm{~h}$ entre la dernière injection de eFSH et l'injection de hCG destinée à induire l'ovulation (Welsh et al 2006). Ce traitement, appelé «coasting», aurait tendance à produire plus d'embryons mais aucun résultat n'est significatif. A l'heure actuelle, il n'est donc pas possible de privilégier l'une ou l'autre de ces hypothèses.

La diminution de la production d'embryons est associée à l'administration trop précoce au cours de la phase folliculaire de LH ou d'une hormone à effet LH comme hCG. Avec chacune des deux hormones, un plus faible nombre d'embryons est récolté par jument : 0,2 dans le lot traité pendant 5 jours avec hCG seule et 0 dans le lot traité avec eFSH vs 0,67 dans le lot témoin non traité (Briant 2004). Ces deux hormones provoquent une montée préovulatoire de progestérone. Comme proposé chez la vache, cette sécrétion trop précoce de progestérone peut perturber la rencontre entre l'ovocyte et les spermatozoïdes. Une autre hypothèse démontrée dans l'espèce humaine est envisageable : les femmes présentant des lutéinisations prématurées, au cours des traitements de superovulation, ont des chances de succès en fécondation in vitro moindres (Shulman et al 1996, Bosch et al 2003). La progestérone, sécrétée trop précocement agirait sur l'endomètre, en perturbant la fenêtre d'implantation (Kolb et Paulson 1997). 
Les effets potentiels des traitements de superovulation sur la maturation ovocytaire ont été recherchés chez la jument. Le seul résultat qui irait dans ce sens rapporte un plus faible taux de récupération des ovocytes, par follicule aspiré après traitement avec l'EPE (21 à $26 \%$, par rapport à des cycles témoins (35\% à $79 \%$ ) (Scoggin et al 2002). Les auteurs ont attribué ces faibles taux à une immaturité des follicules. Mais, les études portant sur la qualité des ovocytes n'ont montré aucun effet, ni sur la maturation nucléaire après administration de pFSH (Folltropin $\AA$ ) (Brück et al 1997) ou de eFSH, ni sur la maturation cytoplasmique après administration de eFSH (Bézard et al 1995). Cependant dans ce dernier cas, les auteurs avaient récupéré les ovocytes, chez les femelles témoins comme chez les femelles traitées, $35 \mathrm{~h}$ après une injection I.V. d'EPE, censée reproduire le pic périovulatoire de LH. Il est donc possible que cette injection ait uniformisé la maturation ovocytaire dans les deux lots.

L'effet de la dépression ou de la suppression du pic de LH sur la production d'embryons, n'a pas chez la jument des conséquences aussi défavorables que chez la vache. En effet, chez la jument poney Welsh recevant 1,72 $\mathrm{mg}$ de eFSH par jour en 3 injections I.V., les paramètres de la réponse au traitement ont été comparés entre les juments avec et sans pic de LH (figure 6, Briant 2004). Les juments sans pic étaient celles qui avaient le plus grand nombre de follicules préovulatoires (4,6 vs 2,2 pour les juments avec pic), sécrétant de plus grandes quantités d'œstrogènes, susceptibles de déprimer la LH. Ces juments avaient également plus d'ovulations $(3,8$ vs 2 pour les juments avec pic) et plus d'embryons (1,6 vs 0,6 pour les juments avec pic). La suppression du pic de LH est donc plutôt la conséquence d'une stimulation efficace que le témoin d'une perturbation indésirable. Cette observation peut être rapprochée du fait que, chez la jument non stimulée, la suppression du pic périovulatoire de LH par administration d'un antagoniste de GnRH, n'a d'effet indésirable, ni sur l'ovulation, ni sur la sécrétion du corps jaune, ni sur la production d'embryons (Briant et al 2003).

Figure 6. Profils plasmatiques moyens de LH chez des juments non traitées $n=10$ ou traitées avec eFSH 1,72 mg/jour en 3 injections IV $n=10$. Les juments traitées ont été séparées en 2 groupes : avec $(n=5)$ ou sans pic $(n=5)$ de LH endogène (Adapté de Briant 2004).

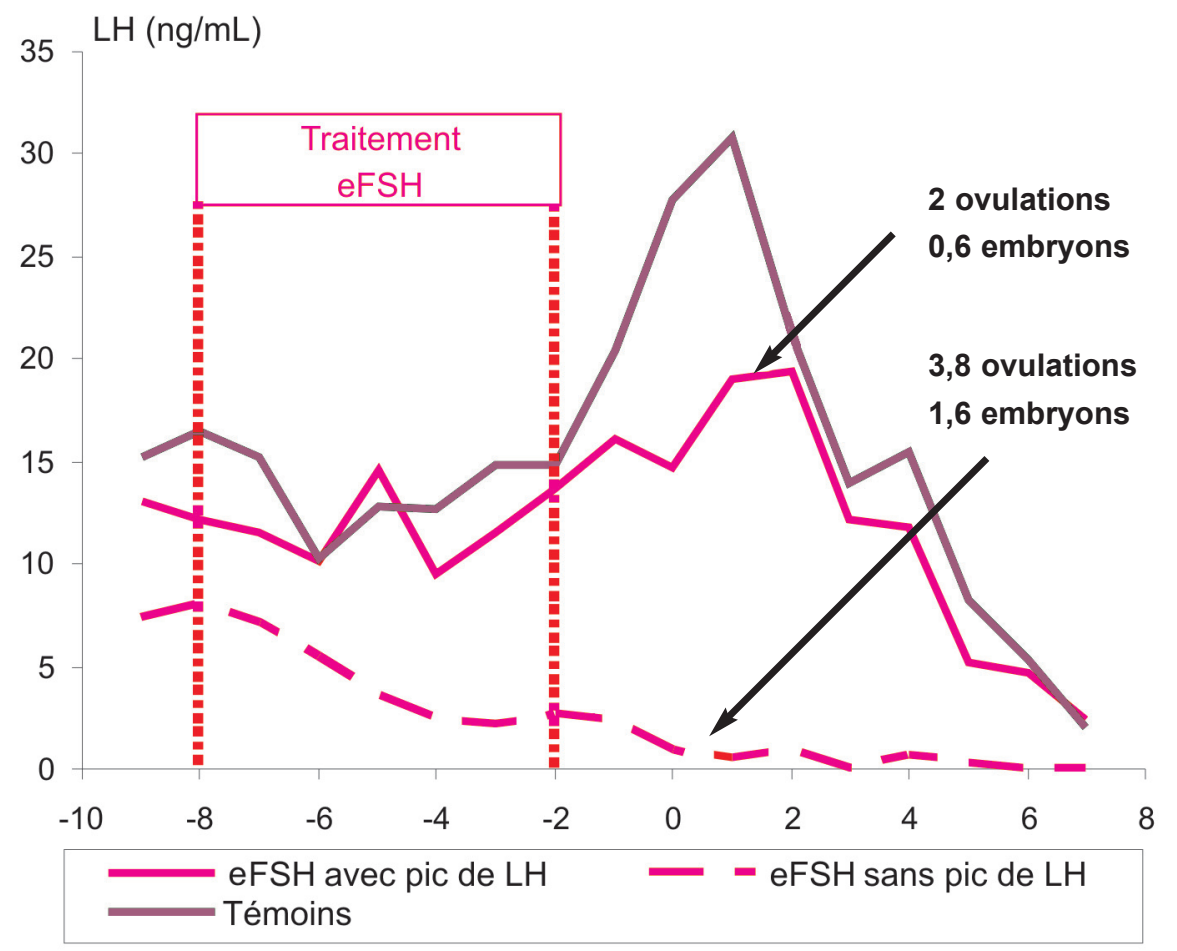

Jours (J0 = jour de l'ovulation)

Les données sont présentées depuis le lendemain de l'injection de prostaglandine F2 $\alpha$ et J0 est le jour de l'ovulation. La période de traitement est comprise entre les lignes pointillées rouges.

- Sur ces bases une posologie idéale a été recherchée

Des études récentes ont recherché la posologie de eFSH capable de reproduire des taux plasmatiques «physiologiques» de FSH, susceptibles d'induire une superovulation sans effets indésirables (Briant et al 2004b). Chez la jument cyclique non stimulée, l'ovulation simple est la conséquence de la dépression de $\mathrm{FSH}$, provoquée par la dominance folliculaire. L'hypothèse de départ était donc que l'administration quotidienne d'une quantité de eFSH, correspondant à celle produite par une femelle ovariectomisée, et donc sans rétrocontrôle ovarien, serait adéquate pour induire une stimulation ovarienne. Les concentrations plasmatiques moyennes de FSH, ont donc été mesurées chez quatre ponettes Welsh ovariectomisées, puis les paramètres pharmacocinétiques de l'hormone ont été déterminés chez ces mêmes ponettes, soumises à un traitement pour déprimer la FSH endogène.

Chez les ponettes ovariectomisées, les concentrations plasmatiques moyennes de FSH sont comprises entre 28 et $42 \mathrm{ng} / \mathrm{mL}$, le temps moyen de résidence de la eFSH entre 5,8 et $10,6 \mathrm{~h}$ et la clairance plasmatique entre 0,22 et $0,31 \mathrm{~mL} \cdot \mathrm{kg}^{-1} \cdot \mathrm{mn}^{-1}$. Le taux de production moyen de $\mathrm{FSH}$ a donc été estimé en moyenne à $3,2 \pm 0,3 \mathrm{mg} /$ jour le taux maximal étant de $\overline{3}, 45 \mathrm{mg} /$ jour. A titre de comparaison, chez la jument entière cyclique, ces taux sont compris entre 0,84 et $2,6 \mathrm{mg} /$ jour (Irvine 1979).

Sur cette base, trois posologies décroissantes de eFSH ont été comparées : $6,6 \mathrm{mg} / \mathrm{jour}$ (dose 2 : approximativement 2 fois la dose 1), $3,45 \mathrm{mg} /$ jour (dose $1=$ taux de production journalier maximal) et $1,72 \mathrm{mg} /$ jour (dose 1/2). La eFSH a été administrée par voie I.V., 3 fois par jour, afin d'obtenir une biodisponibilité maximale et tenir compte du temps moyen de résidence de l'hormone. L'ensemble des résultats est présenté figures 7A et 7B (Briant et al 2005).

La comparaison des paramètres de la réponse ovarienne entre les cycles stimulés et non stimulés (figure 7A) montre que : la stimulation ovarienne (nombre de follicules préovulatoires /cycle, moyenne $\pm \mathrm{ET}$ ) varie peu en fonction de la dose de eFSH injectée : $3,7 \pm 0,9($ dose 2$), 3,4 \pm 1,5$ (dose 1$)$ et $(3,3 \pm 2,1)($ dose $1 / 2)$. L'augmentation des quantités de eFSH ne permet donc pas d'augmenter la stimulation qui 
Figure 7A. Paramètres de la réponse ovarienne (moyenne + écart type) chez des juments poneys Welsh traitées avec eFSH ( $n=10 /$ lot) à différentes doses déterminées par rapport au taux de production de la ponette ovariectomisée.

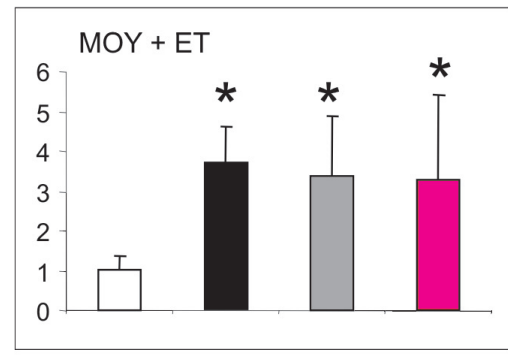

Nb follicules préovulatoires/jument

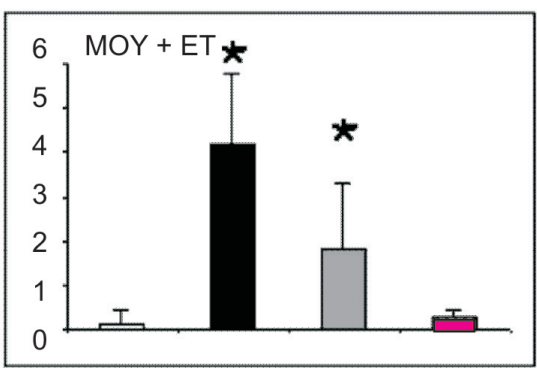

$\mathrm{Nb}$ follicules anovulatoires/jument

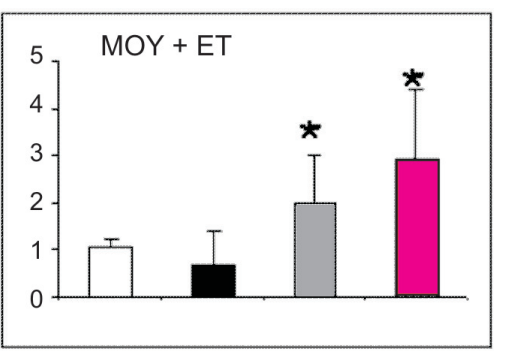

Nb ovulations/jument

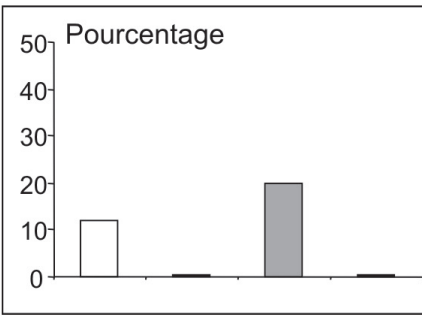

Pourcentage de juments avec ovulation précoce

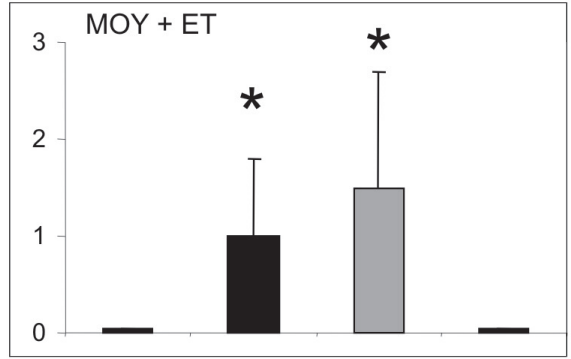

Nb follicules lutéinisés/jument

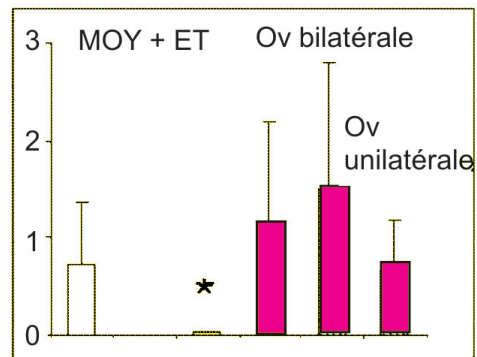

Nb embryons/jument

Témoin (moyenne des trois expériences)

Dose 6,6 mg/jour trois injections IV

Dose $3,45 \mathrm{mg} /$ jour trois injections IV = Taux de production de la ponette ovariectomisé

Dose $1,72 \mathrm{mg} / \mathrm{jour}$ trois injections IV

* : différence significative par rapport au lot témoin $(P<0,05)$ (Briant et al 2005).

atteint un maximum compris entre 3 et 4 follicules préovulatoires. Avec les deux doses les plus élevées, le nombre d'ovulations est inférieur au nombre de follicules préovulatoires $(0,7 \pm 0,7$ (dose 2) et $2 \pm 1$ (dose 1)) du fait de la formation de follicules lutéinisés $(1 \pm$ $0,8$ (dose 2$)$ et $1,5 \pm 1,2($ dose 1$)) \overline{\text { et }}$ anovulatoires $(4,2 \pm \overline{1}, 6($ dose 2$)$ et 1,8 $\pm 1,5($ dose 1$))$. Les données individuelles montrent que les nombres de follicules préovulatoires et d'ovulations ne sont pas corrélés. De plus, avec la dose 1 , des ovulations précoces sont constatées (20\% des cycles). Au contraire avec la dose $1 / 2$, le nombre d'ovulations $(2,9 \pm 1,5)$ est très voisin du nombre de follicules préovulatoires $(3,3+2,1)$ et les deux paramètres sont corrélés $(\mathrm{R}=0,98)$. Il n'y a pas ou peu de follicules lutéinisés $(0)$, anovulatoires $(0,3 \pm 0,4)$ et d'ovulations précoces $(0)$. Cette absence de formation de structures ovariennes indésirables avec la dose $1 / 2$ est associée à une tendance à l'augmentation du nombre moyen d'embryons $(1,1 \pm 1,1)$, qui est plus nette en cas d'ovulations bilatérales $(1,5 \pm 1,3)$.
La comparaison des données plasmatiques endocriniennes (figure 7B) montre qu'avec les deux plus fortes doses, des modifications importantes sont induites. Avec la $1 / 2$ dose, les concentrations plasmatiques de FSH et d'oestrogènes pendant le traitement ne sont que modérément augmentées. La seule altération concerne le pic périovulatoire de LH qui est déprimé. Après ovulation, les concentrations de progestérone sont très significativement augmentées ce qui correspond au fait que pratiquement tous les follicules préovulatoires ont ovulé et se sont transformés en corps jaunes actifs.

L'apport d'une quantité quotidienne de eFSH égale à environ $50 \%$ du taux de production de la ponette ovariectomisée (soit $150 \%$ de celui de la ponette entière), sans apport excessif de $\mathrm{LH}$, permet d'obtenir une stimulation ovarienne satisfaisante et une tendance à l'augmentation de la production d'embryons. Cette posologie évite la formation de structures ovariennes anormales. Elle permet aussi la restauration des profils endocriniens, sauf la dépres- sion du pic périovulatoire de LH, indissociable de l'augmentation des oestrogènes induite par la stimulation folliculaire, mais qui n'a pas d'effet indésirable.

Cependant, la voie d'administration I.V. et le nombre quotidien d'injections appliqués dans cette série d'expériences ne sont pas faciles à mettre en oeuvre en pratique. Ces résultats permettent cependant de comprendre pourquoi le fractionnement de la dose quotidienne de eFSH est préférable. Dans ces conditions, si une certaine proportion de perte est acceptée, le traitement préconisé depuis peu, par voie I.M. avec 2 injections quotidiennes, peut être conseillé.

c) De plus, les pertes en embryons par rapport au nombre d'ovulations peuvent être attribuées à d'autres causes qu'une posologie inadaptée.

- Les pertes en embryons seraient plus importantes après ovulations unilatérales qu'après ovulations bilatérales. 
Figure 7B. Paramètres de la réponse endocrinienne (moyenne + erreur standard) chez des juments poneys Welsh traitées avec eFSH $(n=10 /$ lot) à différentes doses déterminées par rapport au taux de production de la ponette ovariectomisée.
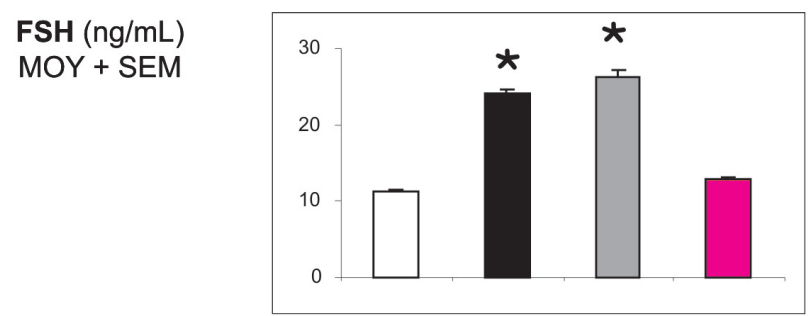

Concentration moyenne/jour/ponette pendant le traitement
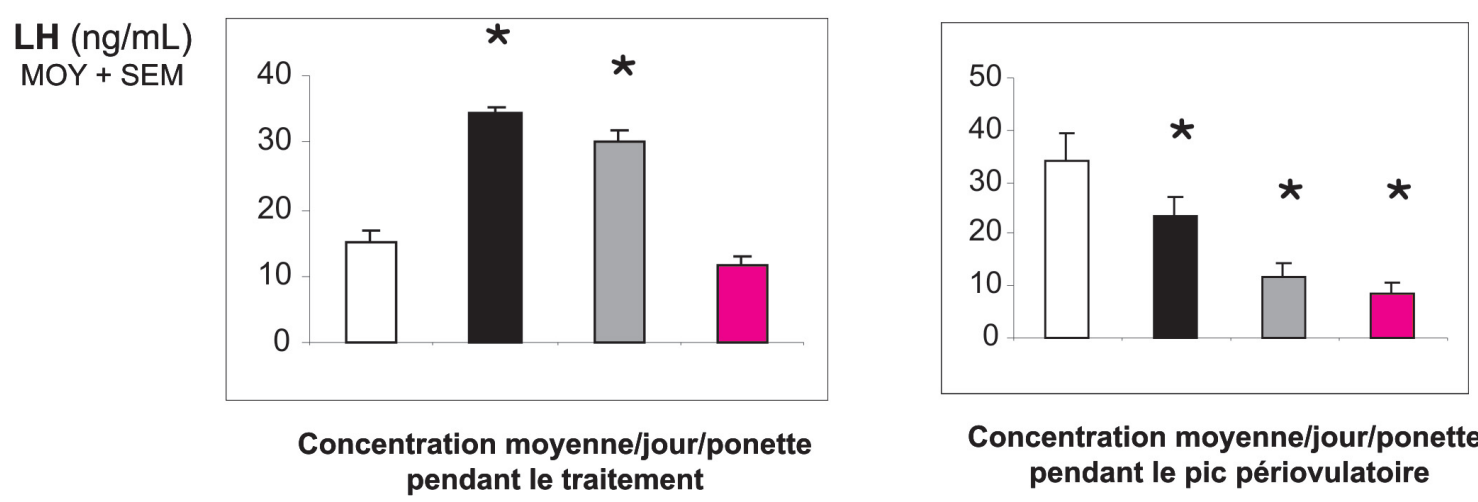

Concentration moyenne/jour/ponette pendant le pic périovulatoire
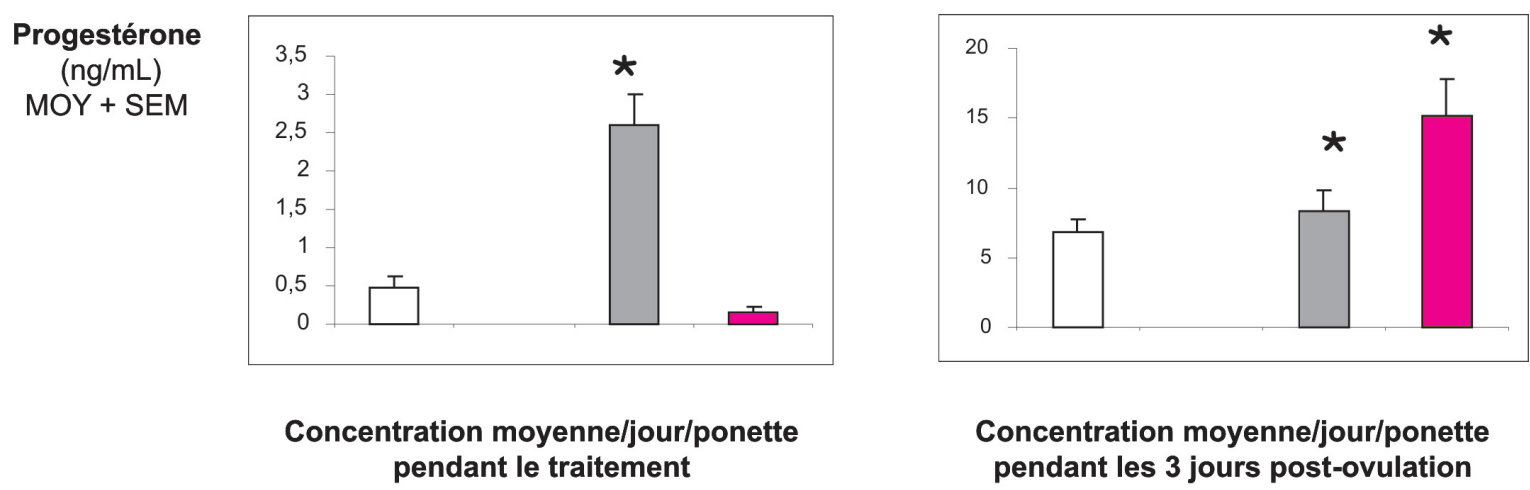

Concentration moyenne/jour/ponette pendant les 3 jours post-ovulation
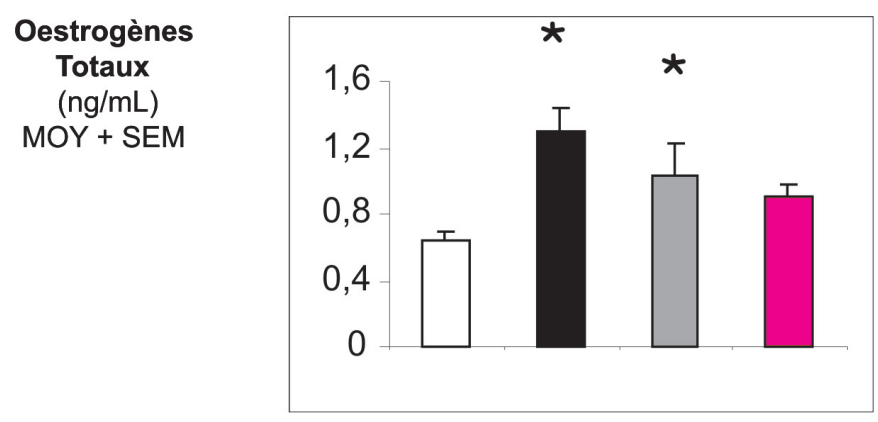

Concentration moyenne/jour/ponette pendant le traitement

Témoin (moyenne des trois expériences)

Dose 6,6 mg/jour trois injections IV

Dose $3,45 \mathrm{mg} /$ jour trois injections IV = Taux de production de la ponette ovariectomisé

Dose $1,72 \mathrm{mg} / \mathrm{jour}$ trois injections IV

* : différence significative par rapport au lot témoin $(P<0,05)$ (Briant et al 2005). 
Plusieurs auteurs rapportent la tendance selon laquelle, après traitement de superovulation, les polyovulations bilatérales (sur les 2 ovaires) seraient plus fertiles que les polyovulations unilatérales (Ginther 1989, Palmer et Hajmeli 1992, Briant et al 2004). Ainsi, dans un troupeau de ponettes Welsh suivi pendant plusieurs années, lors de superovulation induite, le nombre moyen d'embryons récoltés par cycle, 7 jours après ovulation, est de 1,2 $\pm 0,3$ en cas d'ovulations bilatérales et de 0,7 $\pm 0,2$ en cas d'ovulations unilatérales. Cet effet est également connu en cas de polyovulation naturelle au cours de cycles non stimulés car dans le même troupeau au cours de cycles non stimulés, le nombre d'embryon par cycle est de 1,03 \pm 0,13 en cas de polyovulation bilatérale et de 0,59 $\pm 0,01$ en cas de polyovulation unilatérale (Briant 2004). Cet effet est donc indépendant du traitement de superovulation. Il pourrait s'agir soit d'une gêne mécanique à l'expulsion des ovocytes lors de l'ovulation ou à la descente des ovocytes ou des embryons, soit d'un effet autocrine inhibiteur des follicules. lées

- D'autres hypothèses ont été formu-

Certains auteurs ont suggéré qu'en cas de superovulation tous les ovocytes ne seraient pas capables de migrer jusqu'à la fosse ovulatoire (Lapin et Ginther 1977), mais cette hypothèse est controversée (Dippert et al 1994). D'autres ont recherché les embryons et les ovocytes non fécondés par lavage des oviductes, 2 jours après ovulation, chez des juments traitées avec l'EPE (Dippert et al 1994) et ont conclu que le traitement n'induisait pas de perte d'ovocytes ou d'embryons, tout du moins dans les 2 jours post-ovulation. Ces mêmes auteurs ont suggéré que le traitement induirait une altération du transit des embryons vers l'utérus, entre 2 jours ( 2 embryons/cycle en moyenne) et 7 jours $(1,2)$ post ovulation. Cependant, les deux expériences n'étant pas contemporaines et les méthodologies différentes ces résultats ne peuvent être comparés (Dippert et al 1994). Enfin, les expériences ayant étudié la qualité des embryons obtenus après superovulation par transfert dans des juments receveuses sont contradictoires (Woods et Ginther 1983a, Squires et al 1987).

\section{3 / L'ensemble des données disponibles permet actuel- lement de proposer le protocole de traitement sui- vant}

Synchronisation des cycles. Avant application du traitement de superovulation, une synchronisation des cycles avec un progestagène, éventuellement associé à l'œstradiol, peut être effectuée (Palmer 1985, Pierson et Ginther 1990). Cependant, les auteurs n'en ont pas étudié les effets spécifiques sur la stimulation ovarienne ou la production d'embryons.

L'administration de PGF2 $\alpha$ quelques jours avant le début du traitement avec eFSH a tendance à diminuer le nombre de jours de traitement et à augmenter le nombre moyen d'ovulations (Woods et Ginther 1983b). Depuis peu (Niswender et al 2003, Mc Cue et al 2006, Raz et al 2006), l'administration de PGF2 $\alpha$ est effectuée après deux jours de traitement comme chez les bovins. Cette technique donne de bons résultats mais n'a pas été démontrée meilleure que la précédente.

Début et fin du traitement. Le traitement doit être commencé avant l'expression de la dominance folliculaire qui induit l'atrésie des follicules. Ce sont les follicules de plus de $10 \mathrm{~mm}$ de diamètre au début du traitement qui seront stimulés par le traitement (Woods et Ginther 1985). Cependant la présence d'un follicule de plus de $25 \mathrm{~mm}$ au début du traitement n'aurait pas d'effet défavorable ni sur le nombre d'ovulations, ni sur le nombre d'embryons (Squires et al 2006). En pratique, le traitement peut être commencé soit après 7 jours de phase lutéale, soit quand la croissance folliculaire a démarré sur un follicule dominant de $20-25 \mathrm{~mm}$ (Squires et al 2006). L'administration plus précoce du traitement, dès le début de la phase lutéale, n'améliore pas la stimulation (Palmer et Hajmeli 1992).

Dans tous les cas, il est indispensable de suivre la croissance folliculaire par échographie transrectale afin de connaître la taille du follicule dominant au début du traitement, suivre jour après jour l'évolution de la vague folliculaire stimulée et déterminer le moment de l'arrêt du traitement. En effet, le traitement est poursuivi jusqu'au stade préovulatoire avancé, c'est à dire quand les gros follicules attei- gnent $35 \mathrm{~mm}$. L'ovulation est alors induite par administration d'hormone à effet LH. Pour tenter de limiter la désensibilisation du follicule aux gonadotrophines, certains auteurs ont appliqué la méthode de «coasting» décrite cidessus, sans que la production d'embryons soit significativement augmentée (Welsh et al 2006).

Afin de reproduire un environnement endocrinien plus physiologique, certains auteurs ont essayé d'appliquer des protocoles inspirés des espèces de rente. L'administration d'EPE en doses décroissantes n'améliore pas les résultats: en moyenne 5 ovulations et 1,8 embryons (Machado et al 2004). Il en est de même du traitement associant 3,5 jours de traitement avec eFSH ( $25 \mathrm{mg}$ en 2 injections) puis $12,5 \mathrm{mg}$ de LH jusqu'à l'induction d'ovulation, qui produit en moyenne 3,5 ovulations et 1,9 embryons (Welsh et al 2006).

Enfin, certains auteurs ont testé des traitements très courts afin de réduire un éventuel effet nocif induit par le traitement. La eFSH a été injectée au moment où les diamètres des deux plus gros follicules étaient compris entre $17 \mathrm{~mm}$ et $25 \mathrm{~mm}$. Ce traitement a été comparé avec le traitement dit long, où la eFSH était injectée depuis l'administration de PGF2 $\alpha$ jusqu'à l'induction d'ovulation (Bézard et al 1995). Les ovocytes ont ensuite été ponctionnés au stade préovulatoire après injection I.V. d'EPE (effet LH). Parmi les 46 ovocytes collectés au total, 6 étaient immatures dont 5 obtenus après le traitement court. Il apparaît donc préférable de poursuivre le traitement jusqu'au moment de l'induction des ovulations.

L'induction de l'ovulation avec une hormone à activité LH permet une meilleure synchronisation des ovulations (Woods et Ginther 1983b). L'administration de 3300 UI d'hCG à des juments traitées avec l'EPE, permet d'induire les ovulations multiples le même jour chez $100 \%$ des juments ayant reçu hCG (vs $25 \%$ des juments non traitées avec hCG). Chez les juments non traitées la première et la dernière ovulation sont séparées de 1,6 $\pm 0,4$ jours. De plus, hCG semble plus efficace que le Deslorelin (Ovuplant ${ }^{\circledR}$ ) pour induire les ovulations. En effet dans une expérience comparant les 2 molécules, les auteurs ont montré qu'avec hCG tous les follicules préovulatoires $(3,4)$ ovulent, alors qu'avec le Deslorelin moins de la moitié de ces 
follicules $(3,8)$ ovulent $(1,8)$ (Niswender et al 2003). Le traitement peut être reconduit au cours de plusieurs cycles successifs, sans effets additionnels des cycles (Lapin et Ginther 1977) et ce, au cours d'une même saison, sur une période de 6 mois (Palmer 1985).

\section{4 / Différentes stratégies pourraient être développées pour améliorer l'efficacité des traitements}

Certaines de ces stratégies sont utilisées en pratique courante dans l'espèce humaine, d'autres ont été testées dans les espèces de rente avec toutefois de résultats contradictoires, d'autres enfin sont proposées à partir de résultats préliminaires obtenus dans l'espèce équine.

\section{1 / Assurer un meilleur contrô- le des évènements avec le GnRH, ses agonistes ou ses anta- gonistes}

Dans les espèces de rente, l'augmentation ou l'amélioration de la qualité du recrutement est possible, grâce à des prétraitements utilisant des agonistes (McNeilly et Fraser 1987) ou des antagonistes de GnRH (Cognié 1999, Lopez-Alonzo et al 2005). Par exemple, chez la brebis laitière de race Lacaune, un traitement avec un antagoniste de GnRH (antarelix) pendant 10 jours avant le début de la stimulation gonadotrope, assure une augmentation du nombre d'embryons transférables et d'agneaux nés d'environ $50 \%$ (Cognié 1999). Comme les traitements de synchronisation avec les progestagènes, ce type de protocole doit permettre en théorie d'obtenir le recrutement d'une population de follicules sains après suppression de tous les follicules atrétiques ou déjà dominants. Actuellement, ces traitements ne sont utilisés qu'à titre expérimental car aucune molécule n'a obtenu d'autorisation de mise sur le marché pour les espèces de rente.

Dans l'espèce humaine, les programmes de reproduction assistée associent les gonadotrophines avec, soit un agoniste de GnRH (Filicori et al 1996) soit un antagoniste (Reissmann et al 1995), afin de maîtriser au mieux la dose de gonadotrophines actives et éviter les pics de LH précoces, responsables de la «perte» de cycles. Ces traitements visent à obtenir une augmentation modérée du nombre de follicules préovulatoires, tout en limitant au maximum le nombre de follicules de petite ou moyenne taille, et éviter le syndrome d'hyperstimulation (Filicori et Cognigni 2001). Les agonistes sont utilisés pendant une période de traitement de 7 à 20 jours. Après un effet stimulant sur la sécrétion de LH pendant $12 \mathrm{~h}$, ils exercent ensuite une action désensibilisante de plus longue durée sur les récepteurs hypophysaires. Ils permettraient une amélioration du nombre et de la qualité des embryons par rapport à des cycles non traités (Chetkowski et al 1989, Abdalla et al 1990, Polson et al 1991, Tummon et al 1992). Les antagonistes sont utilisés pendant une période de traitement plus courte de 2,5 à 7 jours. Ils n'induisent pas de désensibilisation des récepteurs et leurs effets sont donc quasiment limités à la durée $\mathrm{du}$ traitement. Les ovocytes et embryons obtenus seraient de meilleure qualité qu'avec les agonistes (Diedrich et al 1994, Minaretzis et al 1995, Felberbaum et al 1996, Engel et al 2002, Hwang et al 2003).

Chez la jument, quelques essais ont été effectués avec des agonistes de GnRH. L'administration d'un implant de Buséréline le jour de l'ovulation (durée de relarguage de 28 jours) n'apporte pas d'amélioration globale ni en nombre d'ovulations, ni en nombre d'embryons (Dippert et al 1992). Une grande variabilité inter animaux quant aux effets «inhibiteurs» de l'agoniste, en est peut être responsable, cette dernière pouvant être attribuable à la forme d'administration sous-cutanée. Un traitement comparable avec implants de Deslorelin (deux implants de 2,1 mg, Ovuplant $\AA$ ) posés avant l'ovulation du cycle précédent, n'a pas non plus d'effet améliorateur sur la réponse ovarienne à l'EPE, en terme de nombre d'ovocytes collectés par jument (1,8 sans agoniste vs 1,3 avec agoniste) ou de proportion d'ovocytes récupérés par follicule ponctionné ( $25 \%$ et $21 \%$ respectivement) (Scoggin et al 2002). Cette proportion est très décevante par rapport aux autres espèces.

Les effets d'antagonistes n'ont pas encore été testés. Or ceux-ci sont connus, mais au cours de cycles non stimulés (Guillaume et al 2002, Briant et al 2003, 2004a). L'administration d'antarelix (Teverelix $\left.{ }^{\circledR}\right)$ chez la jument poney Welsh, à raison de $300 \mu \mathrm{g} / \mathrm{kg}$ en deux injections I.V. quotidiennes, pendant au moins trois jours, permet d'interrompre la croissance de la vague folliculaire en cours, pendant le temps du traitement. A l'arrêt du traitement une nouvelle vague folliculaire est recrutée et une ovulation est observée environ deux semaines plus tard. La fertilité sur cette vague retour est satisfaisante. Sur ces bases, pour obtenir une efficacité maximale du traitement de synchronisation, il apparaîtrait préférable d'attendre que la croissance folliculaire ait redémarré spontanément avant d'administrer le traitement de superovulation.

\section{2 / Augmenter le recrutement folliculaire et favoriser la fécon- dation et le développement embryonnaire par un prétraite- ment avec la GH}

Dans les espèces domestiques de rente, des essais d'utilisation de la GH (ou somatotropine) ont été effectués en association avec les traitements de superovulation. Certains résultats semblent intéressants mais sont parfois contradictoires. Ces traitements auraient deux types d'actions.

Administrés avant le traitement de superovulation, le plus souvent à raison d'une injection de préparation à effet retard, ces traitements permettraient d'augmenter la population de petits follicules recrutés susceptibles de répondre au traitement de superovulation. Par exemple, chez la génisse, un prétraitement de $\mathrm{GH}$ bovine recombinante (bGH) associé à un traitement avec eCG permet d'augmenter le nombre moyen d'ovulations ( 23 vs 12,5 avec eCG seule) et le nombre d'embryons $(7,4$ vs 3,8), mais pas le nombre d'embryons transférables (Gong et al 1993). Dans une autre expérience, les mêmes auteurs obtiennent une tendance à l'augmentation du nombre d'embryons transférables $(3,6$ vs 2,9) (Gong et al 1993).

Au contraire, un traitement associant bGH et FSH chez le buffle d'eau n'a pas d'effet sur le nombre de corps jaunes (6 vs 4,3 chez les animaux témoins), d'embryons récoltés par collecte $(4,5$ vs 2,3$)$, mais permet d'augmenter le nombre d'embryons transférables $(3$ vs 0,8$)$ ainsi que la proportion d'embryons transférables (75 vs 33\%) (Songsasen et al 1999).

Chez la brebis un prétraitement avec la bGH n'augmente pas la réponse au traitement de superovulation. (Eckery et al 1994). Cependant, dans ce cas précis, l'hormone utilisée n'est pas celle de l'espèce.

Le deuxième type d'action de la GH, démontré plus récemment, s'effectue- 
rait au niveau de la fécondation et du développement embryonnaire. En effet, chez la vache, l'administration de bGH au moment de l'insémination artificielle augmente le pourcentage d'embryons transférables et stimule le développement embryonnaire, jusqu'au stade blastocyste chez les femelles receveuses. Les mécanismes d'action ne sont pas élucidés (Moreira et al 2002). Ce traitement a été testé dans un programme commercial de transfert embryonnaire, associé à la FSH, mais n'a pas amélioré la réponse ovarienne (nombre de corps jaunes) ou le nombre d'embryons transférables. Les auteurs ont attribué ce résultat décevant au fait qu'il s'agissait de traitements répétés au cours de cycles successifs (Hasler et al 2003). Chez la brebis, ce traitement permettrait d'augmenter le nombre moyen d'embryons transférables $(3,9$ vs 1,7) ainsi que le nombre d'agneaux nés par femelle (2,28 vs 0,84 ). Ces effets bénéfiques seraient attribués, soit à une action directe sur la maturation ovocytaire soit à un effet indirect sur l'environnement de l'oviducte (Folch et al 2001).

Chez la jument, un traitement associant 1'EPE avec la GH porcine n'améliore pas la réponse ovarienne (Hofferer et al 1991). Mais dans cette expérience les conditions n'étaient pas optimales, car l'hormone utilisée n'était pas d'origine équine et le traitement avait probablement été effectué trop tard dans le cycle. Des travaux en cours dans notre laboratoire avec la $\mathrm{GH}$ équine (Guillaume et al 2006b) ont pour objectif de maîtriser l'action physiologique de cette hormone, afin de pouvoir l'utiliser dans ce type de traitement.

\section{3 / Déterminer si l'état corpo- rel a une incidence sur la répon- se aux traitements de superovu- lation}

Comme décrit dans le paragraphe 2.1, chez la vache, l'augmentation des apports nutritionnels module certains paramètres de la réponse au traitement de superovulation. Cependant, tous les mécanismes en jeu ne sont pas élucidés et les réponses pas encore bien maîtri- sées. Des études récentes menées chez la jument laissent supposer que l'état corporel pourrait avoir une incidence sur la réponse aux traitements de superovulation. Ainsi, une diminution importante de la ration chez la jument poney Welsh (voir $\S 2.1$ ) a des conséquences sur la croissance folliculaire, avec diminution du volume total des follicules tout au long de la phase folliculaire et diminution de la proportion de juments présentant des doubles ovulations (Guillaume et al 2006a). Il serait donc approprié de comparer les effets d'un traitement de superovulation sur des juments maigres et grasses.

\section{4 / Augmenter le recrutement avec les anticorps anti-inhibine}

L'immunisation anti-inhibine permet d'obtenir une stimulation folliculaire, en bloquant le rétrocontrôle négatif de l'inhibine sur les gonadotrophines et en stimulant donc la production de FSH endogène. Dans les différentes espèces de rente cette technique n'a été utilisée qu'au plan expérimental. Chez la jument, l'immunisation passive (injection d'anticorps anti-inhibine) induit une stimulation folliculaire uniquement au cours du cycle traité avec obtention de 1,35 (Briant 2004) à 4 ovulations (Nambo et al 1998) en moyenne par cycle. De plus, au cours d'une expérience où des juments poneys Welsh ont été suivies au cours de 3 cycles successifs : témoin (injection de sérum physiologique), traité (injection d'anticorps anti-inhibine) et post-traitement (injection de sérum physiologique), le nombre de petits follicules recrutés a été significativement augmenté au cours du cycle post-traitement par rapport aux deux autres $(7,7$ vs 4,85 et 4,7$)$ (Briant 2004). Cependant ces petits follicules recrutés surnuméraires n'ont pas évolué jusqu'au stade préovulatoire. Par contre, l'administration d'un traitement de superovulation aurait peut être pu prendre le relais jusqu'au stade préovulatoire. Ce type d'effet n'a pas été rapporté jusqu'à présent dans une autre espèce animale. Il faut reconnaître cependant la lourdeur considérable de ce type de protocole pour la production et la purification de sérums immuns.

\section{Conclusions et perspectives}

Dans l'espèce équine, la variabilité des réponses aux traitements de superovulation est donc attribuable à des causes multiples parmi lesquelles l'utilisation de posologies mal adaptées : dose totale, contamination en $\mathrm{LH}$, fractionnement de la dose journalière. Ces facteurs interviennent largement dans les échecs rencontrés au cours des précédentes décennies. Les études récentes permettent maintenant de proposer des traitements qui peuvent être considérés efficaces, avec l'espérance d'obtenir pour une jument une moyenne de 3 à 4 ovulations et 1,8 à 2,5 embryons par cycle traité.

Cependant dans cette espèce, le problème de disponibilité des gonadotrophines utilisées est crucial. En effet, il n'est pas possible d'utiliser celles commercialisées pour les ruminants. La nécessité de préparer la FSH équine à partir d'hypophyses prélevées en abattoir impose de disposer d'un circuit d'approvisionnement constant. Or, la fourniture des hypophyses est limitée et centralisée sur certains pays ayant de gros troupeaux équins (Etats-Unis, Canada, Amérique du Sud). De plus, l'implication d'un laboratoire qui prendrait en charge une demande d'AMM pour ce type de produit reste incertaine, en termes de rentabilité, dans la mesure où le produit ne serait utilisable que pour une seule espèce. Enfin, l'obtention de nos jours d'une AMM pour un produit préparé à partir d'extraits de cerveaux est très improbable, pour des raisons sanitaires. Dans ces conditions, seule la production d'une hormone recombinante est envisageable ce qui permettrait de résoudre les problèmes de disponibilité et de qualité sanitaire. Cette possibilité est tout à fait réaliste, puisque très récemment, une $\mathrm{LH}$ recombinante équine simple chaîne a été produite et a montré une activité à la fois chez l'étalon (Jablonka-Shariff et al 2007) et la jument (Yoon et al 2006). Toutefois, la prise en charge de la mise sur le marché de ce type de produit par un laboratoire pharmaceutique reste actuellement peu probable.

\section{Références}

Abdala H., Ahuja K., Leonard T., Morris N., Honour J., Jacobs H., 1990. Comparative trial of luteinizing hormone releasing hormone analog human menopausal gonadotropin and clomiphene citrate human menopausal gonadotropin in an assisted conception program. Fertil. Steril., 53 (3), 473-478.

Alvarenga M.A., Mc Cue P.M., Bruemmer J., Neves Neto J.R.,Squires E.L., 2001. Ovarian superstimulatory response and embryo pro- duction in mares treated with equine pituitary extract twice daily. Theriogenology, 56, 879887.

Bézard J., Goudet G., Duchamp G., Palmer E., 1995. Preovulatory maturation of ovarian follicles 
and oocytes in unstimulated and superovulated mares. Biol. Reprod. Monogr., serie 1, 261-271.

Bosch E., Valencia E., Escudero E., Crespo J., Simon C., Remohi J., Pellicer A., 2003. Premature luteinization during gonadotropin releasing hormone antagonist cycles and its relationship with in vitro fertilization outcome. Fertil. Steril., 80 (6), 1444-1449.

Bradford G., Quirke J., Hart R., 1971. Natural and induced ovulation rate of Finnish landrace and other breeds of sheep. Anim. Prod., 13, 627-635.

Briant C., 2004. Stimulation ovarienne chez la ponette Welsh en vue de la production d'embryons. Limites physiologiques et techniques. Thèse de Doctorat, Université de Tours, sciences de la vie, 281p.

Briant C., Ottogalli M., Morel M., Guillaume D., 2003. Use of a GnRH antagonist, antarelix, associated or not with hCG, to control ovulation in cyclic pony mares. Domest. Anim. Endocrinol., 24, 305-322.

Briant C., Ottogalli M., Guillaume D., 2004a. Attempt to Control of the day of ovulation in cyclic pony mares by associating a GnRH antagonist with hCG. D Domest. Anim. Endocrinol., 27, 165-178.

Briant C., Toutain P.L., Ottogalli M., Magallon T., Guillaume D., 2004b. Kinetic studies and production rate of eFSH in ovariectomised pony mares. Application to the determination of a dosage-regimen for eFSH in a superovulation treatment. J. Endocrinol., 182, 43-54.

Briant C., Blanc M., Toutain P.L., Ricard A., Guillaume G., 2005. Superovulation chez la jument : quel avenir ? 31 emes Journ. Rech. Equine, 2 mars. Les Haras Nationaux, Paris, France. 83-92.

Brück I., Synnestvedt B., Greve T., 1997. Repeated transvaginal oocyte aspiration in unstimulated and FSH-treated mares. Theriogenology, 47, 1157-1167.

Brück I., Bézard J. Baltsen M., Synnestvedt B., Couty I., Greve T., Duchamp G., 2000. Effect of administering a crude equine gonadotrophin preparation to mares on follicular development, oocyte recovery rate and oocyte maturation in vivo. J. Reprod. Fertil., 118, 351-360.

Byrne B., Fowler P.A., Fraser M., Culler M., Templeton A., 1995. GnSAF bioactivity in serum from superovulated women is not blocked by inhibin antibody. Biol. Reprod., 52, 88-95.

Callesen H., Greve T., Hyttel P., 1986. Preovulatory endocrinology and oocyte maturation in superovulated cattle. Theriogenology, 25, 71-86.

Chetkowsky R., Kruse L., Nass T., 1989. Improved pregnancy outcome with the addition of leuprolide acetate to gonadotropins for in vitro fertilization. Fertil. Steril., 52 (2), 250-255.

Cognié Y., 1999. State of the art in sheep-goat embryo transfer. Theriogenology, 51 (1), 105116.

David J., 1975. A survey of eggs in the oviducts of mares. J. Reprod. Fertil., Suppl. 23, 513-517.

De Loos F.A., Bevers M.M., Dielman S.J., Kruip T., 1991. Follicular and oocyte maturation in cows treated for superovulation. Theriogenology, 35, 537-546.

Diedrich K., Diedrich C., Santos E., Zoll C., Al-Hasani S., Reissmann T., Krebs D., Klingmüller D., 1994. Suppression of the endogenous luteinizing hormone surge by the gonadotrophin releasing hormone antagonist
Cetrorelix during ovarian stimulation. Hum. Reprod., 9 (5), 788-791.

Dippert K.D., Hofferer S., Palmer E., Jasko D.J., Squires E.L., 1992. Initiation of superovulation in mares 5 or 12 days after ovulation using equine pituitary. Theriogenology, 38, 695-710.

Dippert K.D., Jasko D.J., Seidel G.E., Squires E.L., 1994. Fertilization rates in superovulated and spontaneously ovulating mares. Theriogenology, 41, 1411-1423.

Donaldson L., 1985. LH and FSH profiles at superovulation and embryo production in the cow. Theriogenology, 23, 441-447.

Douglas R., 1979. Review of induction of superovulation and embryo transfer in the equine. Theriogenology, 11, 33-79.

Driancourt M.A., 2001. Regulation of ovarian follicular dynamics in farm animals. Implications for manipulation of reproduction. Theriogenology, 55, 1211-1239.

Eckery D.C., Moeller C.L., Nett T.M., Sawyer H.R., 1994. Recombinant bovine somatotropin does not improve superovulatory response in sheep. J. Anim. Sci., 72 (9), 2425 2430.

Engel J.B., Ludwig M., Felberbaum R., Albano C., Devroey P., Diedrich K., 2002. Use of Cetrorelix in combination with clomiphene citrate and gonadotrophins: a suitable approach to friendly IVF. Hum. Reprod., 17 (8), 2022-2026.

Evans M.J., Gastal E.L., Silva L.A., Gastal M.O, Kitson N.E., Alexander S.L., Irvine C.H.G., 2006. Plasma LH concentration after administration of human chorionic gonadotropin to estrous mares. Anim. Reprod. Sci., 94, 191194.

Felberbaum R., Reissmann T., Küpker W., AlHasani S., Bauer O., Schill T., Zoll C., Diedrich C., Diedrich K., 1996. Hormone profiles under ovarian stimulation with human menopausal gonadotropin (hMG) and concomitant administration of the gonadotropin releasing hormone (GnRH) antagonist Cetrorelix at different dosages. J. Assist. Reprod. Genet., 13 (3), 216222.

Filicori M., Cognigni G.E., 2001. Roles and novel regimen of luteinizing hormone and follicle stimulating hormone in ovulation induction. J. Clin. Endocrinol. Metabol., 86, 1437-1441.

Filicori M., Cognigni G.E., Arnone R. Carbone F., Falbo A., Tabarelli C., Ciampaglia W., Casadio P., Spettoli D., Pecorari R., 1996. Role of different GnRH agonist regimens in pituitary suppression and the outcome of controlled ovarian hyperstimulation. Hum. Reprod., 11 (3), 123-132.

Folch J., Ramon J.P., Cocero M.J., Alabart J.L., Beckers J.F., 2001. Exogenous growth hormone improves the number of transferable embryos in superovulated ewes. Theriogenology, 55 (9), 1777- 1785.

Foote R., Ellington J., 1988. Is a superovulated oocyte normal? Theriogenolgy, 29, 111-123.

Fowler P.A., Sorsa-Leslie T., Harris W., Mason H.D., 2003. Ovarian gonodotrophin surge attenuating factor (GnSAF): where are we after 20 years of research? Reproduction, 126, 689-699.

Ginther O.J., 1989. Twin embryos in mares: I. from ovulation to fixation. Equine Vet. J., 21, 166-170

Ginther O.J., 1992. Characteristics of the ovulatory season. In: Reproductive biology of the mare: basic and applied aspects. Library of congress catalog cross plains Equiservices Publishing (Ed) 173-232

Ginther O.J.,Pierson R.A., 1989. Regular and irregular characteristics of ovulation and the interovulatory interval in mares. J. Equine Vet. Sci., 9, 4-12.

Ginther O.J., Douglas R.,Lawrence J.R., 1982. Twinning in mares: A survey of veterinarians and analyses of theriogenology records. Theriogenology, 18, 333-347.

Gong J.G., Bramley T.A., Wilmut I.,Webb $\mathrm{R}, 1993$. Effect of recombinant bovine somatotropin on the superovulatory response to pregnant mare serum gonadotropin in heifers. Biol. Reprod., 48, 1141-1149.

Gong J.G., Armstrong D.G., Baxter G., Hogg C.O., Garnworthy P.C.,Webb R., 2002. The effect of increased dietary intake on superovulatory response to FSH in heifers. Theriogenology, 57 (6), 1591-1602.

Gonzalez A., Wang H., Carruthers T.D. Murphy B.D., Mapletoft R.J., 1994. Increased ovulation rates in PMSG-stimulated beef heifers treated with a monoclonal PMSG antibody. Theriogenology, 41, 1631-1642.

Greve T., Callesen H., 2001. Rendez-vous in the oviduct: implications for superovulation and embryo transfer. Reprod. Nutr. Dev., 41, 451459.

Guillaume D., Bruneau B., Briant C., 2002. Comparison of the effects of two GnRH antagonists on LH and FSH secretion, follicular growth and ovulation in the mare. Reprod. Nutr. Dev. 42, 251-264.

Guillaume D., Salazar-Ortiz J., Martin-Rosset W., 2006a. Effects of nutrition level on mares' ovarian activity and on horses' puberty. In: Nutrition and feeding of the broodmare. $\mathrm{N}$. Miraglia, W. Martin-Rosset (Ed). EAAP Scient. Series, Wageningen Academic Publisher Wageningen, The Netherland, 315-339.

Guillaume D., Chesneau D., Popot M.A., Leveau M., Salazar-Ortiz J., 2006b. Taux circulants de l'hormone de croissance chez le cheval

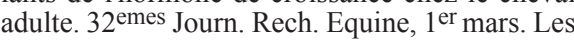
Haras Nationaux, Paris, France, 39-50.

Guillou F., Combarnous Y, 1983. Purification of equine gonadotropins and comparative study of their acid-dissociation and receptor-binding specificity. Biochim. Biophys. Acta, 755, 229 236.

Hansen P.J., Drost M., Rivera R.M., PaulaLopes F.F., al-Katanani Y.M., Krininger C.E., Chase C.C.J., 2001. Adverse impact of heat stress on embryo production: causes and strategies for mitigation. Theriogenology, 55 (1), 91-103.

Hasler J.F., Bilby C.R., Collier R.J., Denham S.C., Lucy M.C., 2003. Effect of recombinant bovine somatotropin on superovulatory response and recipient pregnancy rates in a commercial embryo transfer program. Theriogenology, 59 (9), 1919-1928.

Henry M., Coryn M., Vandelplassche M., 1982. Multiple ovulation in the mare. Zentralblatt Veterinamedizin Reihe A, 29, 170-184.

Hofferer S., Duchamp G., Palmer E., 1991. Ovarian response in mares to prolonged treatment with exogenous equine pituitary gonadotrophins. J. Reprod. Fertil., Suppl. 44 341-349.

Hofferer S., Lecompte F., Magallon T., Palmer E., Combarnous Y., 1993. Induction of ovulation 
and superovulation in mares using equine $\mathrm{LH}$ and FSH separated by hydrophobic interaction chromatography. J. Reprod. Fertil., 98, 597-602.

Humblot P., Rodrigues J.L., Nibart M., Silvestrini Tiezzi F.L., Jeanguyot N., Thibier M., 1994. Effet du mode de synchronisation des cycles sexuels sur la réponse hypophysaire et la fonction ovarienne après superovulation chez la vache. Elev. Ins., 261, 7-18.

Hwang J., Huang L., Hsieh B., Tsai Y., Huang S., Chen C., Hsieh M., Chen P., Lin Y., 2003. Ovarian stimulation by clomiphene citrate and $\mathrm{hMG}$ in combination with cetrorelix acetate for ICSI cycles. Hum. Reprod., 18 (1), 45-49.

Hyttel P., Callesen H., Greve T., 1986. Ultrastructural features of preovulatory oocyte maturation in superovulated cattle. J. Reprod. Fertil., 76, 645-656.

Irvine C.H.G., 1979. Kinetics of gonadotrophins in the mare. J. Reprod. Fertil., Suppl 27, 131-141

Jablonka-Shariff A., Roser J.F., Bousfield G.R., Wolfe M.W., Sibley L.E., Colgin M., Boime I., 2007. Expression and bioactivity of a single chain recombinant equine luteinizing hormone (reLH). Theriogenology, 67 (2), 311-320.

Jensen A., Greve T., Madej A., Edqvist L., 1982. Endocrine profiles and embryo quality in the PMSG-PGF2 alpha treated cow. Theriogenology, 18, 33-44.

Kafi M., McGowan M.R., 1997. Factors associated with variation in the superovulatory response of cattle. Anim. Reprod. Sci., 48, 137-157.

Kolb B.A., Paulson R.J., 1997. The luteal phase of cycles utilizing controlled ovarian hyperstimulation and the possible impact of this hyperstimulation on embryon implantation Am. J. Obst. Gynecol., 176 (6), 1262-1267.

Lafri M., Ponsart C., Nibart M., Durand M., Morel A., Jeanguyot N., Badinand F., De Mari K., Humblot P., 2002. Influence of CIDR treatment during superovulation on embryo production and hormonal patterns in cattle. Theriogenology, 58, 1141-1151.

Lapin D., Ginther O.J., 1977. Induction of ovulation and multiple ovulations in seasonally anovulatory and ovulatory mares with an equine pituitary extract. J. Anim. Sci., 44, 834-842.

Liu J., Sirois J., 1998. Follicle size-dependant induction of prostaglandin $\mathrm{G} / \mathrm{H}$ synthase- $2 \mathrm{du}-$ ring superovulation in cattle. Biol. Reprod., 58, $1527-1532$.

Lopez-Alonso C., Encinas T., Veiga-Lopez A., Garcia-Garcia R.M., Cocero M.J., Ros J.M., McNeilly A.S., Gonzalez-Bulnes A., 2005. Follicular Growth, endocrine response and embryo yields in sheep superovulated with FSH after pre-treatment with a single short-acting dose of GnRH antagonist. Theriogenology, 64 (8), 1833-1843.

Machado M.S., Carmo M.T., Squires E.L., Roser J.F., Alvarenga M.A., 2004. Follicular dynamic, superovulatory response and embryo production in mares treated with equine pituitary extract and equine FSH. $6^{\text {th }}$ Int. Symp. Equine Embryo Transfer, 4-6 August, Rio de Janeiro Brazil, 29.

Mapletoft R.J., Steward K.B., Adams G.P., 2002. Recent advances in the superovulation in cattle. Reprod. Nutr. Dev., 42, 601-611.

Mc Cue P.M., Squires E.L., 2002. Persistent anovulatory follicles in the mares. Theriogenology, 58, 541-543.
Mc Cue P.M., Logan N.L., Alonso M.A., Squires E.L., 2006. Superovulation of mares: effects of eFSH treatment during consecutive or alternate cycles and as short-term therapy before follicular divergence. Anim. Reprod. Sci., 94, 404-407.

McNeilly A.S., Fraser H.M., 1987. Effect of $\mathrm{GnRH}$ agonist-induced suppression of LH and FSH on follicle growth and corpus luteum function in the ewe. J. Endocrinol., 115, 273-282.

Minaretzis D., Alper M.M., Oskowitz P., Lobel S.M., Mortola J.F., Pavlou S.N., 1995. Gonadotropin releasing hormone antagonist versus agonist administration in women undergoing controlled ovarian hyperstimulation: cycle performance and in vitro steroidogenesis of granulosa lutein cells. Am. J. Obst. .Gynecol., 172, 1518-1525.

Moor R., Kruip A., Green D., 1984. Intraovarian control of folliculogenesis: limits to superovulation. Theriogenology, 21, 103-116.

Moreira F., Badinga L., Burnley C., Thatcher W.W., 2002. Bovine somatotropin increases embryonic development in superovulated cows and improves post-transfer pregnancy rates when given to lactating recipient cows Theriogenology, 57 (4), 1371-1387.

Nambo Y., Kaneko H., Nagata S., Oikawa M. Yoshihara T., Nagamine N., Watanabe G., Taya K. 1998. Effect of passive immunization against inhibin on FSH secretion, folliculogenesis and ovulation rate during the follicular phase of the estrous cycle in mares. Theriogenology, 50, 545-557.

Nibart N., Slimane N., Herrera R., Jeanguyot N., Mechekour F., Humblot P., Thibier M., 1988. Variations des concentrations plasmatiques des hormones gonadotropes $(\mathrm{FSH}, \mathrm{LH})$ et stéroïdes (œstradiol 17 bêta, progestérone) après différents traitements de superovulation chez la vache. Elev. Ins., 226, 11-30.

Niswender K.D., Alvarenga M.A., McCue P., Hardy Q.P., Squires E.L., 2003. Superovulation in cycling mares using equine follicle stimulating hormone (eFSH). Equine Vet. Sci., 23 (11), 497-500.

Osborne V.E., 1966. An analysis of the pattern of ovulation as it occurs in the annual reproductive cycle of the mare in Australia. Aust. Vet. J., $42,149-154$

Palmer E., 1985. Recent attempts to improve synchronisation of ovulation and to induce superovulation in the mare. Equine Vet. J. Suppl. 3, 11-18

Palmer E., Hajmeli G., 1992. Essais de supervulation chez la jument. Rec. Méd. Vét. Spécial Reproduction des équidés, 168, 897-905.

Picton H.M., McNeilly A.S., 1991. Evidence to support of follicle stimulating hormone threshold theory for follicle selection in ewes chronically treated with gonadotrophin-releasing hormone agonist. J. Reprod. Fertil., 93, 43-51.

Pierson R.A., Ginther O.J., 1990. Ovarian follicular response of mares to an equine pituitary extract after suppression of follicular development. Anim. Reprod. Sci., 22, 131-144.

Polson D., MacLachlan V., Krapez J., Wood C., Healy D., 1991. A controlled study of gonadotropin releasing hormone agonist (buserelin acetate) for folliculogenesis in routine in vitro fertilization patients. Fert. Steril., 56 (3), 509514.

Ponsart C., Marquant-Leguienne B., Humblot P., 2004. Les biotechnologies de l'embryon bovin, évolution et perspectives. Renc, Rech, Rum., 11, 361-368.

Price C.A., 1995. Superovulatory treatments do not alter pulsatile LH secretion in ovariectomized cattle. Theriogenology, 43, 543-549.

Price C., Carrière P., Gosselin N., Kohram H., Guilbault L.A., 1998. Effects of superovulation on endogenous LH secretion in cattle, and consequences for embryo production. Theriogenology, $51,37-46$

Putney D.J., Drost M., Thatcher W.W., 1988. Embryonic development in superovulated dairy cattle exposed to elevated ambient temperature between days 1 to 7 post insemination. Theriogenology, 30, 195-209.

Rao M.C., Richards J.S., Midgley A.R. Reichert L.E., 1977. Regulation of gonadotropin receptors by luteinizing hormone in granulosa cells. Endocrinology, 101, 512-523.

Raz T., Gray A., Hunter B., Card C., 2006. Effect of equine follicle stimulating hormone (eFSH) on pregnancy rate and embryo development in mares. Anim. Reprod. Sci., 94, 400-403.

Reissmann T., Felberbaum R., Diedrich K., Engel J., Comaru-Schally A.M., Schally A.V., 1995. Development and applications of luteinizing hormone releasing hormone antagonists in the treatment of infertility: an overview. Hum. Reprod., 10 (8), 1974-1981.

Remy B., Bruyas J.F., Beckers J.F., Leduc F. Fiéni F., Tainturier D., 1997 Ovulation rates in donor mares treated with purified equine FSH. Theriogenology, 47, 394.

Roberge S., Rieger D., Rawlings N., 1995. Periovulatory LH, FSH and steroid hormone profiles in superovulated and unstimulated Holstein heifers. Theriogenology, 44, 59-70.

Robinson J.J., Ashworth C.J., Rooke J.A. Mitchell L.M., McEvoy T.G., 2005. Nutrition and fertility in ruminant livestock. Anim. Fd Sci. Technol., 126 (3-4), 259-276.

Saumande J., 1980. Concentrations of luteinizing hormone, oestradiol 17 beta and progesterone in the plasma of heifers treated to induce superovulation. J. Endocrinol., 84, 425437.

Scoggin C.F., Meira C., McCue P.M. Carnevale E.M., Nett T.M., Squires E.L., 2002. Strategies to improve the ovarian response to equine pituitary extract in cyclic mares. Theriogenology, 58, 151-164.

Shulman A., Ghetler Y, Beyth Y, Ben-Nun I, 1996. The significance of an early (premature) rise in plasma progesterone in in vitro fertilization cycles induced by a «long protocol» of gonadotropin releasing hormone analogue and human menopausal gonadotropines. J. Assist. Reprod. Genet., 13 (3), 207-211.

Songsasen N., Yiengvisavakul V., Buntaracha B., Pharee S., Apimeteetumrong M., Sukwongs Y., 1999. Effect of treatment with recombinant bovine somatotropin on responses to superovulatory treatment in swamp buffalo (Bubalus bubalis). Theriogenology, 52 (3), 377-384

Squires E., McKinnon A., Carnevale E. Morris R., Nett T., 1987. Reproductive characteristics of spontaneous single and double ovulating mares and superovulated mares. J. Reprod. Fertil. Suppl. 35, 399-403.

Squires E.L., Logan N., Welch S., Mc Cue P.M., 2006. Factors affecting the response to administration of equine FSH. Anim. Reprod. Sci., 94, 408-410. 
Takagi M., Kim I., Izadyar F., Hyttel P., Bevers M., Dieleman S., Hendriksen P., Vos P., 2001. Impaired final follicular maturation in heifers after superovulation with recombinant human FSH. Theriogenology, 121, 941-951.

Tummon I., Daniel S., Kaplan B., Nisker J., Yuzpe A., 1992. Randomized, prospective comparison of luteal leuprolide acetate and gonadotropins versus clomiphene citrate and gonadotropins in 408 first cycles of in vitro fertilization. Fertil. Steril., 58 (3), 563-568.
Welch S.A., Denniston D.J., Hudson J.J., Bruemmer J.E., Mc Cue P.M., Squires E.L., 2006. Exogenous eFSH, follicle coasting, and $\mathrm{hCG}$ as a novel superovulation regimen in mares. J. Equine Vet. Sci., 26 (6), 262-270.

Wesson J.A., Ginther O.J., 1981. Influence of season and age on reproductive activity in pony mares on the basis of a slaughterhouse survey. J. Anim. Sci., 52, 119-129.

Woods G., Ginther O.J., 1983a. Intrauterine embryo reduction in the mare. Theriogenology, 20, 699-706.
Woods G., Ginther O.J., 1983b. Induction of multiple ovulations during the ovulatory season in mares. Theriogenology, 20, 347-355.

Woods G., Ginther O.J., 1985. Follicular dynamics in mares treated with an equine pituitary extract. Theriogenology, 23, 297-308.

Yoon M.J., Boime I., Colgin M., Niswender K.D., King S.S., Alvarenga M., Roser J.F., 2006. Single-chain recombinant eLH: induction of ovulation in mares. Anim. Reprod. Sci., 94, 215-216.

\section{Résumé}

Chez les espèces domestiques de rente, les traitements de superovulation sont destinés à augmenter le taux naturel d'ovulations, afin de produire plusieurs embryons au cours d'un même cycle et de les transférer chez des femelles receveuses. Pour l'espèce équine, aucun traitement n'est commercialisé car, d'une part, les gonadotrophines commercialisées pour les autres espèces de rente sont inefficaces chez la jument, d'autre part, les réponses aux traitements montrent une grande variabilité.

Chez la jument, les traitements administrés utilisent la FSH équine (eFSH). La variabilité des réponses concerne à la fois les capacités de stimulation, représentées par le nombre de follicules préovulatoires, le nombre d'ovulations et le nombre d'embryons transférables. Comme pour l'ensemble des espèces de rente, les causes de cette variabilité sont multiples. Parmi celles-ci une posologie d'administration des gonadotrophines inadaptée est responsable d'anomalies de la folliculogénèse et de perturbations endocriniennes qui conduisent à une diminution de la production d'embryons. Partant des modèles proposés dans l'espèce bovine, de nouvelles études ont été conduites chez la jument afin de déterminer les interactions entre les altérations folliculaires et endocrines et de préciser quels étaient les paramètres agissant sur la production d'embryons. Une posologie idéale a ensuite été recherchée comme étant capable de reproduire des taux plasmatiques physiologiques de FSH, susceptibles d'induire une superovulation, sans effets indésirables. Cette posologie correspond à l'apport d'une quantité quotidienne de eFSH égale à environ $50 \%$ du taux de production journalier de la jument ovariectomisée. A partir de l'ensemble de ces données, un protocole de traitement peut actuellement être proposé, avec l'espérance d'obtenir pour une jument et par cycle traité une moyenne de 3 à 4 ovulations et 1,8 à 2,5 embryons. Différentes stratégies sont ensuite abordées qui pourraient améliorer l'efficacité des traitements.

Cependant la commercialisation future d'une préparation de eFSH pour l'espèce équine est incertaine, du fait de la fourniture limitée des hypophyses nécessaires, des contraintes sanitaires liées à ce type de produit et de la nécessité de préparer un produit spécifique pour cette espèce.

\section{Abstract}

\section{Superovulating mares with gonadotrophins: recent developments and state of the art}

In domestic species the aim of superovulation treatments is to increase natural ovulation rates in order to produce several healthy embryos in a single oestrus cycle and to then transfer these embryos into recipient females. Actually, in equine species, no commercial preparation is available for superovulation. Indeed, gonadotrophins licenced for other species are not effective in mares and responses in mares, to superovulation treatments with equine FSH (eFSH) are heterogeneous.

In mares, superovulation treatments must use eFSH and the heterogeneity of response can adversely affect the number of preovulatory follicles, the number of ovulations and the number of transferable embryos. As for other domestic species, there are multiple reasons for this variability. Among them, unsatisfactory dosage regimens for eFSH that can cause unwanted effects on follicular growth, ovulation and plasma hormone levels and decrease embryo production. In this context, our studies in mares have determined the relationships between impaired follicular growth and modified plasma hormone levels and the factors responsible for the decrease in embryo production. An appropriate dosage regimen for eFSH has been proposed on the basis of FSH plasma levels that can induce superovulation without unwanted side effects. This dosage regimen corresponds to $50 \%$ of the daily production rate of the native hormone in an ovariectomized mare. On the basis of these data, a treatment regimen has been suggested, with the aim of producing 3 to 4 ovulations and 1,8 to 2,5 embryos per oestrus cycle. Finally, several strategies have been suggested which could improve the effectiveness of eFSH treatments in mares.

Nevertheless, the future commercialization of an equine FSH preparation is questionnable because the number of pituitary glands available from slaughterhouses is limited, there are strong health requirements associated with this type of product and this preparation has to be species specific.

BRIANT C., GUILLAUME D., TOUTAIN P.-L., BLANC M.-R., 2007. Superovulation chez la jument avec les hormones gonadotropes : le point sur la situation et nouvelles données. INRA Prod. Anim., 20, 275-294. 
